\title{
Alzheimer's disease-related dysregulation of mRNA translation causes key pathological features with ageing
}

Anshua Ghosh', Keiko Mizuno', Sachin S. Tiwari ', Petroula Proitsi (1)', Beatriz Gomez Perez-Nievas ${ }^{1}$, Elizabeth Glennon', Rocio T. Martinez-Nunez ${ }^{2}$ and K. Peter Giese (1) ${ }^{1}$

\begin{abstract}
Alzheimer's disease (AD) is characterised by $A \beta$ and tau pathology as well as synaptic degeneration, which correlates best with cognitive impairment. Previous work suggested that this pathological complexity may result from changes in mRNA translation. Here, we studied whether mRNA translation and its underlying signalling are altered in an early model of AD, and whether modelling this deficiency in mice causes pathological features with ageing. Using an unbiased screen, we show that exposure of primary neurons to nanomolar amounts of A $\beta$ increases FMRP-regulated protein synthesis. This selective regulation of mRNA translation is dependent on a signalling cascade involving MAPKinteracting kinase 1 (Mnk1) and the eukaryotic initiation factor 4E (elF4E), and ultimately results in reduction of CYFIP2, an FMRP-binding protein. Modelling this CYFIP2 reduction in mice, we find age-dependent A $\beta$ accumulation in the thalamus, development of tau pathology in entorhinal cortex and hippocampus, as well as gliosis and synapse loss in the hippocampus, together with deficits in memory formation. Therefore, we conclude that early stages of AD involve increased translation of specific CYFIP2/FMRP-regulated transcripts. Since reducing endogenous CYFIP2 expression is sufficient to cause key features of $A D$ with ageing in mice, we suggest that prolonged activation of this pathway is a primary step toward AD pathology, highlighting a novel direction for therapeutic targeting.
\end{abstract}

\section{Introduction}

Amyloid- $\beta$ (A $\beta)$ plaques ${ }^{1}$ and phosphorylated tau tangles $^{2}$ are classical histological features found in brains of patients suffering from Alzheimer's disease (AD), a neurodegenerative condition that causes loss of memory and other cognitive impairments in older age ${ }^{3,4}$. The best correlate of memory loss is the progressive degeneration of synapses, which occurs prior to neuronal death ${ }^{5}$. Early changes in $A \beta$ metabolism may result in synaptic degeneration via the production of soluble $A \beta_{1-42}$ oligomers $^{6,7}$, a consequence of excessive amyloidogenic processing of the

\footnotetext{
Correspondence: K. Peter Giese (karl.giese@kcl.ac.uk)

${ }^{1}$ Department of Basic and Clinical Neuroscience, Institute of Psychiatry, Psychology \& Neuroscience, King's College London, De Crespigny Park, London SE5 8AF, UK

${ }^{2}$ Department of Infectious Diseases, Asthma UK Centre in Allergic Mechanisms of Asthma, King's College London, 5th Floor Tower Wing, Guy's Campus, London SE1 9RT, UK
}

amyloid precursor protein (APP) ${ }^{8}$. In cultured neurons, $A \beta_{1-42}$ oligomers have been suggested to locally stimulate the synthesis of APP at synapses, via an mRNA translation-dependent mechanism that involves the RNAbinding protein fragile $\mathrm{X}$ mental retardation protein $(\text { FMRP })^{9,10}$. In this manner, $A \beta_{1-42}$ may drive its own production, resulting in a feed-forward loop of toxicity at a particularly vulnerable location. FMRP binds to hundreds of mRNAs in the brain, including App mRNA ${ }^{11-13}$. Its primary cellular function is thought to be the inhibition of mRNA translation, which requires FMRP-mRNA interaction $^{11,14,15}$, although in some instances FMRP may be involved in both nucleo-cytoplasmic and dendritic mRNA transport ${ }^{16,17}$.

FMRP binds to the highly conserved cytoplasmic FMRP-interacting proteins 1 and 2 (CYFIP1 and 2, also known as Sra- 1 and Pir121, respectively $)^{18}$. Both proteins 
are expressed in various tissues including the brain ${ }^{19}$, where they localise to both excitatory ${ }^{20}$ and inhibitory ${ }^{21}$ synapses of hippocampal neurons. Although little is known about their precise function due to their relatively recent discovery, they have both overlapping and unique functions $^{22,23}$. CYFIP1 is thought to repress capdependent translation of specific mRNAs by interacting with the eukaryotic initiation factor 4E (eIF4E), and CYFIP2 has an identical eIF4E-binding motif to CYFIP $^{22,24}$. In addition to regulating translation of mRNA, CYFIP1 and CYFIP2 are part of the Wiskott-Aldrich syndrome protein-family verprolinhomologous protein (WAVE) complex that regulates actin polymerisation at synapses ${ }^{25-28}$. CYFIP2 protein expression is reduced in post-mortem $\mathrm{AD}$ brain and in an $\mathrm{AD}$ mouse model ${ }^{23}$. Modelling the AD-related reduced CYFIP2 expression in heterozygous Cyfip2 null mutant mice $\left(\right.$ Cyfip $\left.2^{+-}\right)$, which have no overt phenotypes ${ }^{19}$, led to increased APP protein expression, enhanced tau phosphorylation at CaMKII phospho-sites in synapses, elevated soluble $A \beta_{1-42}$ levels, and changes in synapse morphology in the young adult hippocampus ${ }^{23}$.

Taken together, these findings suggested that amyloid accumulation, tau pathology, and synaptic degeneration are due to, at least in part, an $A \beta$-induced elevation of protein synthesis that involves CYFIP2. Here, we tested this hypothesis in primary neuronal cultures and found that nanomolar amounts of $A \beta_{1-42}$ preparations increase net protein synthesis and modulate the association of specific putative FMRP targets with ribosomes. Mechanistically, $A \beta_{1-42}$ causes MAPK-interacting kinase (Mnk1)dependent phosphorylation of eIF4E and dissociation between eIF4E and CYFIP2, ultimately resulting in ubiquitination and reduction of CYFIP2 levels. Since early changes in $\mathrm{A} \beta$ metabolism reduce CYFIP2 expression, we studied the phenotype of heterozygous Cyfip 2 null mutants in older age, as ageing is the biggest risk factor for $\mathrm{AD}$, and homozygous knockouts are not viable ${ }^{19}$. We establish that aged Cyfip 2 heterozygotes develop amyloid and tau pathology, gliosis, spine loss and more severe memory impairment than in young age, indicating that reduction of a single endogenous mouse gene can cause key aspects of AD-type pathology in the mouse brain. Therefore, our work proposes a central role for CYFIP2 in $\mathrm{AD}$ pathogenesis, as a potential modulator of A $\beta$-dependent mRNA translation.

\section{Materials and methods Cyfip2 $^{+/-}$mice}

As detailed previously ${ }^{23}$, Cyfip 2 heterozygous null mutant mice on the C57BL/6N genetic background were obtained from the Wellcome Trust Sanger Institute (Wellcome Trust Genome Campus, Cambridge, UK). Mice designated Cyfip $2^{+/-}$have a Cyfip $2^{\text {tmla(EUCOMM)Wtsi }}$
(ID:33461) allele generated by the European Conditional Mouse Mutagenesis Program which uses a 'knockoutfirst' design ${ }^{19,29}$. Mice were maintained on the C57BL/6N genetic background. Animals were housed on a 12-h light/dark cycle with food and water available ad libitum and genotyped by PCR as described previously ${ }^{23}$. All procedures were undertaken in accordance with the UK Animals (Scientific Procedures) Act 1986. For biochemical studies on younger animals, both male and female mice were used. For behavioural and histological analyses on aged animals, only females were used, including a female-only 3-month-old control group.

\section{Fear-conditioning memory}

All animals used for experiments were handled $2 \mathrm{~min}$ per day either for 3 days (3-4-month old) or 5 days (12month old) before conditioning. All experiments were performed during the light cycle. During training, each mouse was placed into the chamber (Med Associates Inc., St. Albans, VT, USA) in a soundproof box. After a 120-s introductory period, a tone $(75 \mathrm{~dB}, 10 \mathrm{kHz})$ was presented for $30 \mathrm{~s}$, and the mouse received a 2-s foot shock $(0.7 \mathrm{~mA})$ which co-terminated with the tone. After an additional $30 \mathrm{~s}$, the mouse was returned to the home cage. Twentyfour hours after training, the mice were brought back to the conditioning chamber for 5 min to test for contextual fear memory. Freezing behaviour during each 2-s shock was scored every $5 \mathrm{~s}$, blind to genotype.

\section{Immunohistochemistry/immunofluorescence (IHC/IF)}

For histological analyses of aged mice, 12-month-old female Cyfip $2^{+/-}$mutants and wild-type littermates were used. To control for the effects of normal ageing, a group of 3-month-old female wild-type mice also on the C57BL/ $6 \mathrm{~N}$ background were added to the analysis. Mice were anaesthetised with an intraperitoneal injection of Eutha$\mathrm{tal}^{\circledR}$ (Merial, Toulouse, France), and intra-cardially perfused with a $4 \%$ paraformaldehyde solution after an initial PBS wash. Whole brains were removed and stored overnight in 30\% sucrose in PBS, then cut sagittally to separate left and right hemispheres, snap frozen in isopentane, and stored at $-80^{\circ} \mathrm{C}$ until ready for cutting and processing. Brains were mounted onto stages using cryo-embedding compound and coronally cut into $40 \mu \mathrm{m}$ thick sections. For every animal (biological replicate), two sections from the same brain region (technical replicate) were stained per target. Prior to processing, floating sections were washed in TBS, then blocked in $2 \%$ normal goat serum in TBS with $0.1 \%$ Triton X-100 and incubated overnight with primary antibodies diluted in blocking solution. Antibodies used were AT-8 (Thermo Fisher MN1020, 1:100), PHF-1 (Peter Davies, 1:100), tau (Dako A0024, 1:1000), ionised calcium-binding adaptor molecule 1 (Iba1) (Wako Chemicals 019-19741, 1:200), GFAP (Dako 
Z0334, 1:1000), pGSK3ß (Tyr216) (Abcam ab75745, 1:300), 4G8 (Calbiochem NE1002, 1:100). Sections were washed in TBS before incubating in a species-appropriate horseradish peroxidase (HRP)-tagged (IHC) or fluorophore conjugated (IF) secondary antibody, diluted directly in blocking solution.

For IHC, sections were incubated in $2.5 \% \mathrm{v} / \mathrm{v} \mathrm{H}_{2} \mathrm{O}_{2}$ in methanol for $30 \mathrm{~min}$. After secondary antibody incubation sections were washed in TBS and incubated in 3,3'-Diaminobenzidine (DAB) (Sigma) for 2-10 min depending on the antibody used, until the tissue appeared visibly brown, in small batches and blind to group and genotype. Sections were then washed twice in TBS for $10 \mathrm{~min}$, mounted onto glass slides and allowed to dry. Slides were processed for counterstaining of nuclei using haematoxylin. Briefly, mounted sections were gently rinsed with water and then placed in Gill's haematoxylin stain (Vector Laboratories) before being differentiated in $0.5 \%$ acid alcohol, washed, and dehydrated through a series of ethanol solutions $(70 \%, 95 \%, 95 \%, 100 \%, 100 \%)$. Finally, sections were cleared in two changes of xylene before being coverslipped with xylene-based mounting media (Thermo Scientific) using an automated coverslipping system (Thermo Scientific). For IF, slides were coverslipped manually using a fluorescence-compatible mounting medium (Invitrogen ProLong ${ }^{\mathrm{TM}}$ Diamond Antifade mountant with DAPI).

Slides were imaged using the Olympus Slidescanner VS120 with Brightfield or Fluorescence mode. Images were taken with the $40 \times$ objective (NA $0.95,0.17 \mu \mathrm{m} /$ pixel), as automated maximal projections of z-stacks using the maximal density of focal points. Exposure times for fluorescent images were optimised manually prior to the scan, and automatically maintained across sections for the same experiment.

\section{Amytracker $^{\mathrm{TM}}$ staining}

Manufacturer's instructions were used to visualise protein aggregates in mouse brain sections with Amytracker $^{\mathrm{TM}} 680$ (Ebba Biotech). Briefly, sections were rinsed in PBS and fixed in ice-cold $\left(-20^{\circ} \mathrm{C}\right)$ ethanol at RT for $10 \mathrm{~min}$. Tissue sections were rehydrated in a 1:1 mixture of ethanol and water for $5 \mathrm{~min}$ and in PBS for $5 \mathrm{~min}$. Sections were incubated with Amytracker ${ }^{\mathrm{TM}} 680$ dye, diluted 1:1000 in PBS, for $30 \mathrm{~min}$ room temperature. Sections were washed in PBS, mounted onto glass slides with ProLong ${ }^{\mathrm{TM}}$ Diamond (Invitrogen), and coverslipped. Once dry, fluorescence was visualised using the Cy5 filter set of the Olympus Slidescanner.

\section{Dendritic spine analysis}

Brains from 12-month-old female Cyfip $2^{+/-}$mutants and wild-type littermates were used to analyse spine density and morphology and processed for modified
Golgi-Cox impregnation according to manufacturer's instructions (FD Rapid GolgiStain ${ }^{\mathrm{TM}}$ kit, FD NeuroTechnologies, USA). Briefly, brains were isolated as quickly as possible, rinsed in water and immersed in impregnation solutions $\mathrm{A}$ and $\mathrm{B}$ at room temperature for 2 weeks in the dark. Tissue was transferred into Golgi solution $\mathrm{C}$ for $72 \mathrm{~h}$ in the dark at room temperature. Brains were rapidly frozen by dipping into isopentane precooled with dry ice and stored at $-80^{\circ} \mathrm{C}$ until ready for sectioning. Coronal sections of $80 \mu \mathrm{m}$ thickness were cut and mounted on double gelatine-coated slides. Sections were rinsed, stained with solutions $\mathrm{D}$ and $\mathrm{E}$, dehydrated through an ethanol series, and cleared with xylene, before coverslipping with Permount ${ }^{\circledR}$. Slides were allowed to dry overnight before imaging and analysis. Pyramidal neurons in the CA1 region of dorsal hippocampal sections were identified by their triangular soma shape and numerous dendritic spines. A 100× Plan Apo oil-immersion objective (NA 1.40, $0.07 \mu \mathrm{m} /$ pixel) on the Eclipse Ti2 inverted microscope (Nikon) was used to image z-stacks of secondary and tertiary dendrites longer than $10 \mu \mathrm{m}$ in the stratum radiatum (apical dendrites). Fifty dendritic segments were imaged and analysed for each group, and 10-12 cells were imaged per animal. Dendrites were reconstructed in 3D using the Neurolucida 360 system (MBF Bioscience, USA) and dendritic spines were identified and classified as thin, stubby, mushroom or filopodia using parameters based on three-dimensional structures of dendritic spines ${ }^{30}$.

\section{Primary neuronal culture}

Primary cortical neuronal cultures were prepared from Sprague-Dawley rat E18 embryos. Cells were seeded into culture plates coated with $0.2 \mathrm{mg} / \mathrm{mL}$ poly-D-lysine (Sigma) at a density of $\sim 937$ cells $/ \mathrm{mm}^{2}$. Cells were cultured in Neurobasal medium supplemented with $2 \%$ B27, $0.5 \mathrm{mM}$ L-glutamine, and $1 \%$ penicillin/streptomycin (Life Technologies, UK). After 4 days in vitro (DIV), $200 \mu \mathrm{M}$ of D,L-amino-phosphonovalerate (D,L-APV, Abcam) was added to the media to maintain neuronal health over long-term culture and to reduce cell death due to excitotoxicity. Fifty percent media changes were performed weekly until desired time in culture was reached (2728 DIV).

\section{Synthetic $A \beta_{1-42}$ oligomer preparation}

Oligomers from synthetic rat $A \beta_{1-42}$ peptide (Calbiochem) were prepared as detailed ${ }^{31}$. Briefly, a stock solution was prepared at $100 \mu \mathrm{M}$ in $200 \mathrm{mM}$ HEPES (pH 8.5). The solution was gently agitated for $30 \mathrm{~min}$ at room temperature, aliquoted and stored at $-80^{\circ} \mathrm{C}$. Once defrosted for treatments, aliquots were not subjected to any further freeze-thaw. This method of $A \beta_{1-42}$ preparation is thought to form oligomers and not higher 
molecular weight or fibrillar aggregate forms ${ }^{31}$. To verify the composition of the $A \beta_{1-42}$ generated, the NativePAGE $^{\mathrm{TM}}$ (Invitrogen) system was used, and manufacturer's instructions were followed. To confirm the presence of oligomers, $A \beta_{1-42}$ peptides were treated in a 1:1 ratio with guanidine hydrochloride which breaks down oligomers. An antibody raised against the juxtamembrane extracellular domain of $A \beta_{1-42}$, spanning amino acids 17-24 (clone 4G8, Millipore MAB1561, diluted 1:1000), was used for detection of rodent $A \beta_{1-42}$ oligomers. As a control, commonly used human $A \beta_{1-42}$ peptides solubilised either in hexafluoroisopropanol or trifluoroacetic acid were also prepared by removal of solvent and oligomerisation in $\mathrm{PBS}$ at $37^{\circ} \mathrm{C}$ for $3 \mathrm{~h}$ (Supplementary Fig. 1).

\section{Pharmacological treatments}

All treatments were performed directly in cell culture media. Stock solutions of cycloheximide (CHX) (Sigma) and Mnk1 inhibitor compound CGP 57380 (Tocris) in DMSO were diluted to final concentrations in the cell culture medium, using equal volumes of DMSO as control. For oligomeric $A \beta_{1-42}$ treatments, aliquots were defrosted just prior to treatment, and diluted to a final concentration of $100 \mathrm{nM}$ in cell culture medium, using equal volumes of HEPES buffer as control. The surface sensing of translation (SUnSET) assay was used to monitor protein synthesis in cell cultures, following a published protocol ${ }^{32}$. Briefly, puromycin (Sigma) was diluted to a final concentration of $10 \mu \mathrm{g} / \mathrm{ml}$ in culture medium for the last $10 \mathrm{~min}$ of the treatment. Samples were lysed and run as a western blot, using an antipuromycin antibody (Kerafast EQ0001, 1:1000). Prior to blocking and primary antibody incubation, total protein levels on the membrane were detected using the Revert ${ }^{\mathrm{TM}}$ Total Protein Stain Kit (LI-COR) as per manufacturer's instructions.

\section{Cell lysis}

At the end of the treatment culture medium was removed and plates were placed on an ice block. Cells were rinsed briefly with ice-cold PBS, then lysed in equal volumes of lysis buffer $(20 \mathrm{mM}$ Tris $\mathrm{pH} 7.4,150 \mathrm{mM}$ $\mathrm{NaCl}, 1 \%$ Triton X-100, $5 \mathrm{mM}$ EDTA pH 8) supplemented with protease and phosphatase inhibitor cocktails (Sigma), using an ice-cold plastic cell scraper. Lysates were frozen at $-20^{\circ} \mathrm{C}$. Protein amounts were quantified using a BCA kit (Pierce) and proteins were detected by immunoblotting.

\section{Immunoprecipitation}

For immunoprecipitation (IP) in cultured neurons, cells were briefly rinsed in ice-cold PBS, then lysed on ice in cold IP buffer ( $50 \mathrm{mM}$ Tris pH 7.4, $150 \mathrm{mM} \mathrm{NaCl}, 1 \%$ Triton X-100) supplemented with protease inhibitor cocktail (Sigma). For IP in the total mouse hippocampus, frozen tissue was homogenised in the same IP buffer with 20 strokes in a Dounce homogeniser (Smith Scientific). The cell lysate/brain homogenate was centrifuged at $16,089 \times g, 4{ }^{\circ} \mathrm{C}$ for $1 \mathrm{~min}$ and the supernatant used as input for IP. For IP in synaptosomes in mouse hippocampus, crude synaptosomal fractions were freshly prepared, as detailed previously ${ }^{23}$. Protein samples were incubated with pre-cleared magnetic Protein A-coupled Dynabeads $^{\mathrm{TM}}$ (Invitrogen) and primary antibodies against CYFIP1 (Millipore) or CYFIP2 (GeneTex) were added in a 1:50 ratio to the lysate-bead mixture. Tubes were rotated at $4{ }^{\circ} \mathrm{C}$ overnight, spun down briefly, and the supernatant removed using a magnetic rack. Beads were washed $3 \times$ in cold IP buffer, before adding Laemmli sample buffer and boiling at $95^{\circ} \mathrm{C}$ for $10 \mathrm{~min}$. This IP fraction was used fresh for protein detection by immunoblotting. For certain analyses, values were normalised to those of the vehicle control to minimise error between biological replicates. For quantification of protein ubiquitination, cells treated with the proteasomal inhibitor MG132 were used as a positive control to determine the correct molecular weights of ubiquitinated proteins.

\section{Western blotting}

Protein samples were diluted and boiled at $95^{\circ} \mathrm{C}$ in Laemmli sample buffer. In total, $10-20 \mu \mathrm{g}$ of proteins were separated on $4-15 \%$ Criterion $^{\mathrm{TM}} \mathrm{TGX}^{\mathrm{TM}}$ precast gels (Bio-Rad) and then transferred onto a methanol-activated $0.2 \mu \mathrm{m}$ polyvinylidene fluoride membrane (Bio-Rad). Membranes were blocked at room temperature for $1 \mathrm{~h}$ (5\% milk or BSA in TBST pH 7.5) and then incubated in primary antibody diluted in blocking buffer overnight at $4{ }^{\circ} \mathrm{C}$. Primary antibodies were detected using HRPconjugated secondary antibodies (Dako) and chemiluminescent reagent (Thermo Scientific), and signals in the linear range obtained by exposing membranes to X-ray films (Amersham). Prior to probing with other primary antibodies, the membranes were washed in western blot stripping buffer (Santa Cruz Biotechnology). Primary antibodies used were against CYFIP1 (Millipore 07-531, 1:10,000), CYFIP2 (GeneTex GTX124387, 1:10,000), phospho-eIF4E Ser209 (Cell Signalling 9741 1:1000), eIF4E (Cell Signalling 2067, 1:1000), NSE (Millipore AB951, 1:60,000), $\alpha$-synaptotagmin (Sigma S2177, 1:30,000), AT-8 phospho-tau (Invitrogen MN1020, 1:1000), PHF-1 phospho-tau (Peter Davies, 1:1000), total tau (Dako, A0024, 1:10,000) and ubiquitin (Ub) (Millipore 05-1307, 1:1000). Signals were analysed with ImageJ software $(\mathrm{NIH})$ and normalised to appropriate loading controls. 


\section{Ribosomal RNA extraction}

For analysis of ribosome-bound mRNA, primary rat cortical neurons were seeded onto $20 \mathrm{~mm}$ PDL-coated culture dishes at a density of 636 cells $/ \mathrm{mm}^{2}$ and treated with the $A \beta_{1-42}$ preparation at 27 DIV for $24 \mathrm{~h}$. During the last 10 min of treatment on 28 DIV $100 \mu \mathrm{g} / \mathrm{mL} \mathrm{CHX} \mathrm{was}$ added to the culture media to inhibit ribosome drop-off. Once media was removed, cells were placed on a cold block and washed with ice-cold PBS plus $100 \mu \mathrm{g} / \mathrm{mL}$ $\mathrm{CHX}$. Cells were then scraped into ice-cold polysome lysis buffer (10 mM HEPES-KOH (pH 7.4), $5 \mathrm{mM} \mathrm{MgCl}_{2}$, $150 \mathrm{mM} \mathrm{KCl}, 1 \% \mathrm{NP}-40$ ) freshly supplemented with $0.5 \mathrm{mM}$ DTT, $100 \mathrm{U} / \mathrm{mL}$ RNasin RNase inhibitor (Promega), $100 \mu \mathrm{g} / \mathrm{mL} \mathrm{CHX}$, and EDTA-free protease inhibitors (Cell Signalling). The lysate was syringed three times through a 23G needle on ice, then centrifuged at 10,000 $\times$ $g$ for $5 \mathrm{~min}$ at $4{ }^{\circ} \mathrm{C}$. The supernatant was layered onto a $20 \%$ sucrose cushion prepared in the same lysis buffer and then centrifuged at $186,000 \times g$ for $2 \mathrm{~h}$ at $4{ }^{\circ} \mathrm{C}$ in a TLA-55 rotor ultracentrifuge (Beckman Coulter). RNA was isolated from the ribosome-enriched pellet using TRIzol LS reagent according to manufacturer's instructions (Invitrogen).

\section{RNA sequencing and analysis}

Library preparation and RNA sequencing was done by Novogene (Hong Kong). Libraries were made using polyadenylated mRNA isolation, employing NEBNext ${ }^{\circledR}$ Ultra $^{\mathrm{TM}}$ RNA Library Prep Kit for Illumina ${ }^{\circledR}$ (NEB, USA). Three samples had lower RNA amounts and a higher efficiency bead capture was employed for only those, maintaining the same library preparation kit otherwise. Base Quality and Phred score were calculated employing the Illumina CASAVA v1.8 software with more than $92 \%$ of all reads for all samples having a Q30. Bowtie v2.2.3 was used to index the reference genome and trimmed pairedend reads were aligned using TopHat v2.0.12. HTSeq v0.6.1 using the union mode was employed to count mapped reads and generate FPKM (Fragments per Kilobase of transcript sequence) ${ }^{33}$. Differential gene expression analysis was performed employing $\mathrm{DESeq}^{34}$ with Benjamini and Hochberg's FDR correction. Identification of putative FMRP fragments was done by crossreferencing differentially expressed genes between total and ribosome-enriched fractions with previously published datasets ${ }^{13}$ employing R. Heatmap (unsupervised clustering using Pearson), Venn diagram and correlation plot were made using R. All packages are available in CRAN. All RNA sequencing data are deposited in the GEO database with accession number GSE149540.

\section{Statistical analysis}

All experiments were performed at least twice, where possible, and sample sizes selected based on power calculations on preliminary data. All data were analysed using GraphPad Prism version 8. Data were tested for normality with a Shapiro-Wilk test. Data points falling beyond $2 \times \sigma(\mathrm{SD})$ from the mean were considered statistical outliers and removed from every analysis. Statistical significance was determined by two-tailed Student's $t$ test or one-way or twoway analysis of variance (ANOVA) with post-hoc Tukey's correction or Student-Newman-Keuls method. For nonparametric data, Kruskal-Wallis one-way ANOVA with Dunn's testing for multiple comparisons was used. For FMRP analysis a Kolmogorov-Smirnov test was employed to compare cumulative distributions between targets and nontargets; a Fisher's exact two-tailed test was done to determine enrichment of FMRP targets in genes differentially present in ribosomal-enriched fractions. The results having $p<0.05$ were considered significant in every analysis.

\section{Genome-wide association studies (GWAS)}

Single Nucleotide Polymorphisms (SNPs) within $10 \mathrm{~kb}$ upstream and downstream of the coding region of each gene were screened for associations with $\mathrm{AD}$ using the latest genome-wide association and meta-analysis of diagnosed $\mathrm{AD}^{35}$. To account for linkage disequilibrium (LD), tag SNPs were created with the Priority Pruner software (version 0.1.4, http://prioritypruner.sourceforge. net/index.html), which prunes SNPs by removing all those that are in LD with other SNPs in the dataset. The 1000 Genomes (Phase 3) were used as a reference panel for pruning using the Kunkle et al., dataset $p$ values for prioritisation. SNPs with an $r^{2}>0.8$ and within $250 \mathrm{~kb}$ were determined to be in LD. For the selected SNPs, an FDR threshold of 0.05 was applied to correct for multiple testing when looking for associations with $\mathrm{AD}$ ("FDRtools" Rstudio, Version 1.2.1335).

To gain insight into potential regulatory effects of the genetic variants associated with $\mathrm{AD}$ in the five genes, expression quantitative trait loci (eQTL) data from the Genotype-Tissue Expression (GTEx) (Version 6) Project Consortium $^{36}$ and BRAINEAC (www.braineac.org/) ${ }^{37}$ were used to determine whether variants that showed associations with $\mathrm{AD}$ (after correction for multiple testing) affect gene expression as eQTLs. GTEx and BRAINEAC are high-quality databases of matched genotype and gene expression measurements, which enable the quantification of effects of SNPs on gene expression in various tissues, including various brain tissues.

The BRAINEAC eQTLs dataset includes 10 brain regions from 134 neuropathologically normal individuals of European descent. The 10 brain regions are cerebellar cortex, frontal cortex, hippocampus, medulla, occipital cortex, putamen, substantia nigra, temporal cortex, thalamus and intralobular white matter.

The GTEx (version 6) eQTLs dataset includes 53 tissues, 544 donors and 8555 samples. Ten human brain 
tissues were selected including anterior cingulate cortex, caudate basal ganglia, cerebellar hemisphere, cerebellum, cortex, frontal cortex BA9, hippocampus, hypothalamus, nucleus accumbens basal ganglia and putamen basal ganglia, which include at least 70 samples.

\section{Results}

\section{$A \beta_{1-42}$ regulates differential translation of FMRP target} mRNAs in neurons

Previous studies have provided circumstantial evidence that sub-micromolar doses of $A \beta_{1-42}$ may enhance protein synthesis $^{38}$; however, the alternative may be a difference in protein turnover. For the purpose of this study, we selected synthetic rat peptides rather than human peptides. Although it has been suggested that the rodent $A \beta$ sequence is unable to aggregate ${ }^{39,40}$, overexpression of murine $A \beta$ in the mouse brain can result in $A \beta$ accumulation ${ }^{41}$. Therefore, no a priori assumptions were made regarding the amyloidogenic properties of the peptide, to keep the model system as physiological as possible. To directly monitor whether $A \beta_{1-42}$ impacts on protein synthesis, we used the SUnSET assay ${ }^{32}$ which utilises incorporation of puromycin, a structural analogue of aminoacyl tRNAs, into the nascent polypeptide chain, to directly reflect the rate of mRNA translation in vitro. Primary rat cortical neurons were cultured for 27 DIV, before being treated with a preparation of $100 \mathrm{nM}$ synthetic rat $A \beta_{1-42}$ peptides including oligomers (Supplementary Fig. 1) for $24 \mathrm{~h}$, or vehicle as control. Puromycin was added during the last $10 \mathrm{~min}$ of treatment to provide a snapshot of newly synthesised proteins and determine whether $A \beta_{1-42}$ exposure modulates this rate. Cells were lysed and samples subjected to western blot were analysed using an anti-puromycin antibody. When normalised to signal for total protein using Revert ${ }^{\mathrm{TM}}$, we found that $\mathrm{A} \beta_{1}$. 42 treatment led to a significant increase in protein synthesis (Fig. 1a, b). Prior treatment with translational elongation inhibitor $\mathrm{CHX}$ blocked the increase in puromycin signal by $A \beta_{1-42}$, confirming it is translation dependent (Fig. 1a, b). To determine which mRNAs might be regulated at the level of translation by $A \beta_{1-42}$ treatment, we performed paired RNA sequencing in total cytosolic extracts and ribosomal-enriched fractions using sucrose cushions (Fig. 1c) from rat cortical neurons treated for $24 \mathrm{~h}$ with $A \beta_{1-42}$ or vehicle. A minimum of $42 \mathrm{M}$ reads were generated per sample (Supplementary Table 1). Correlation analysis showed that samples cluster differently depending on the subcellular fraction (Supplementary Fig. 2), suggesting that mRNAs pelleted in the sucrose cushion were distinct to total mRNAs. Differential expression between total and ribosomal-bound mRNAs showed 356 unique genes in vehicle-treated cells and 1136 unique genes in $A \beta_{1-42}$-treated cells, with an overlap of 2624 genes (Fig. 1d, Supplementary Table 2).
These data suggest that $A \beta_{1-42}$ exposure modulates the binding of specific mRNAs to ribosomes. We interrogated the presence of previously reported FMRP targets ${ }^{13}$ in these differentially expressed genes and detected FMRP targets in both datasets (Fig. 1e, Supplementary Fig. 3). About 11\% of the differentially ribosome-bound mRNAs were FMRP targets in both vehicle and $A \beta_{1-42^{2}}$-treated cells. However, in $A \beta_{1-42^{-}}$ treated cells there were more unique differentially ribosomebound FMRP targets than in control (Fig. 1e, Supplementary Table 2), suggesting that $A \beta_{1-42}$ exposure may modulate the translation of specific FMRP-bound mRNAs. Unsupervised hierarchical clustering of the identified unique FMRP targets that showed differential binding to ribosomes upon $A \beta_{1-42}$ exposure (not present in vehicle-treated cells) revealed clear groups of genes with differences between vehicle and $A \beta_{1-42}$ treatment and subcellular fraction (Fig. 1f). Biological pathway analysis of these genes with Reactome ${ }^{42}$ was suggestive of pathways enriched in neuronal and synaptic function, such as 'protein-protein interaction at synapses' and 'neurexins and neuroligins' (Supplementary Table 3). Altogether, our data strongly suggest a role for $A \beta$ in the ribosomal association and translation of specific genes modulated by FMRP.

\section{$A \beta_{1-42}$ impacts on protein synthesis via the Mnk/elF4E/ CYFIP axis and reduces CYFIP2 levels}

Since treatment of cortical neurons with $A \beta_{1-42}$ preparations increased protein synthesis and appeared to modulate the translation of specific FMRP-regulated mRNAs (Fig. 1), we further explored the mechanism of this control. CYFIP1 and 2 exist as a part of the FMRP translational repressor complex ${ }^{18}$, while interacting with the translation initiation factor eIF4E which is essential for initiation of cap-dependent mRNA translation in neurons ${ }^{22}$. We hypothesised that the increase in protein synthesis by $A \beta_{1-42}$ may depend on phosphorylation of eIF4E at Ser209, as this phosphorylation is elevated in post-mortem $\mathrm{AD}$ brain $^{43}$ and because it increases translation of specific transcripts ${ }^{44}$. To test this idea, we pharmacologically blocked eIF4E phosphorylation at Ser209 and assessed its impact on $A \beta_{1-42}$-induced protein synthesis in primary neurons (Fig. 2a-d). We used CGP 57380, a competitive inhibitor of MAPK-interacting protein kinase $1(\mathrm{Mnk} 1)^{45}$, a kinase whose only validated substrate is eIF4 $\mathrm{E}^{46,47}$. We found that the $A \beta_{1-42}$ treatment significantly increased eIF4E phosphorylation at Ser209 and the Mnk1 inhibitor blocked this increase (Fig. $2 \mathrm{a}, \mathrm{b})$. Blocking eIF4E phosphorylation prevented an $\mathrm{A} \beta_{1}$ 42 -induced increase in protein synthesis (Fig. 2c, d), suggesting the increased translation by $A \beta_{1-42}$ is dependent on eIF4E phosphorylation at Ser209. Blocking eIF4E phosphorylation did not alter basal protein synthesis (data not shown), in accordance with previous reports ${ }^{48}$.

CYFIP1 represses cap-dependent translation of specific mRNAs by interacting with eIF4E and specifically 

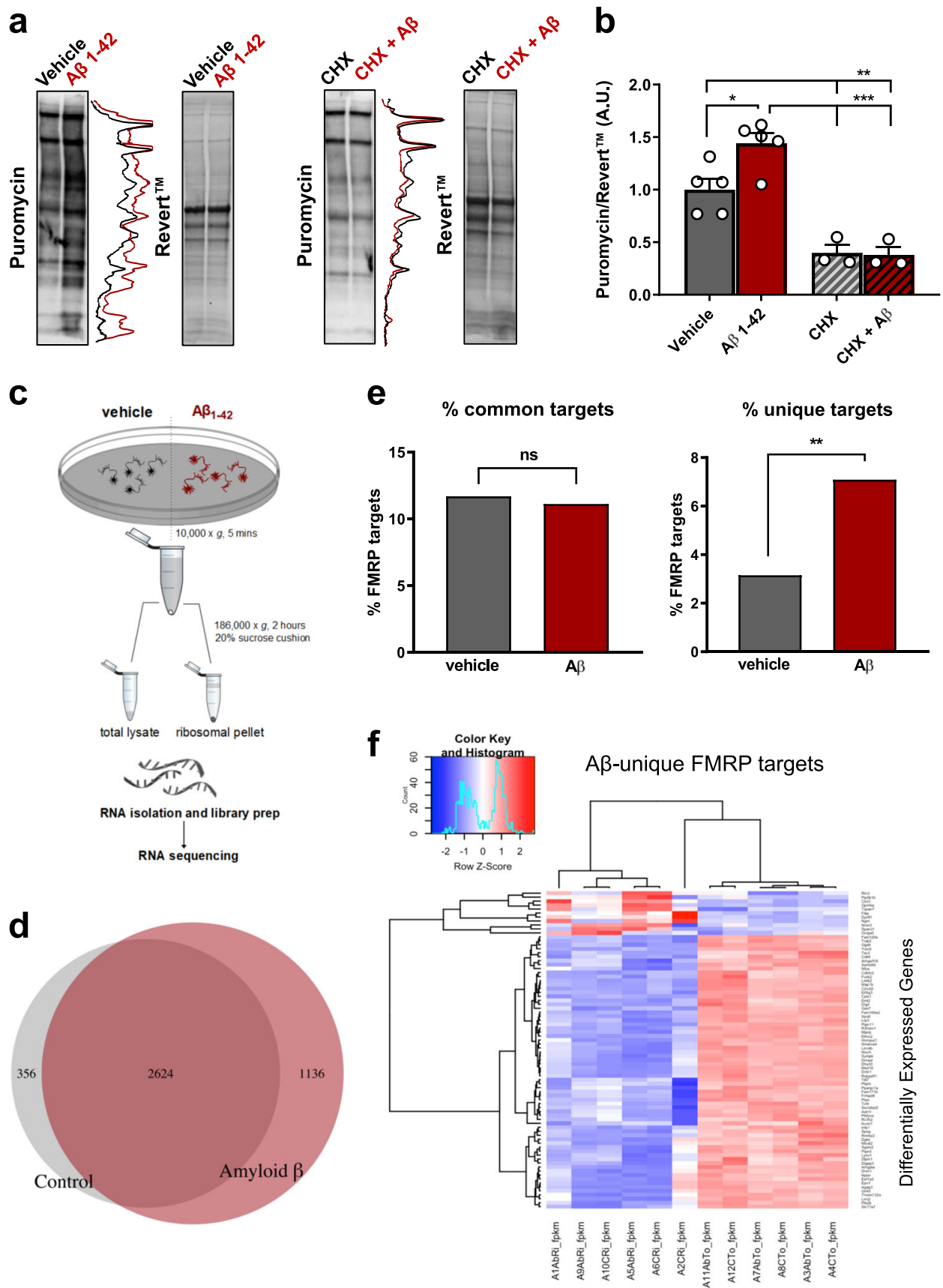

Fig. $1 \mathrm{~A} \beta_{1-42}$ increases protein synthesis in primary cortical neurons by promoting ribosomal association of FMRP-bound mRNAs.

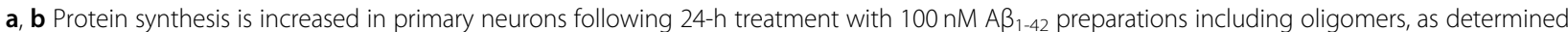
by puromycin levels normalised to Revert ${ }^{\mathrm{TM}}$, a total protein stain $(q=4.88, p<0.05)$. This effect is blocked in the presence of $10 \mu \mathrm{M}$ cycloheximide $(\mathrm{CHX})(q=0.14, p=0.99)$. $\mathbf{c}$ Schematic describing the protocol used to prepare ribosome-enriched pellets from primary neurons for RNA sequencing. d Overlap between mRNA transcripts that are differentially bound to ribosome (versus total) within vehicle- and $A \beta_{1-42}$-treated groups. e Enrichment of FMRP targets in vehicle vs A $\beta$ cells. When considering all putative FMRP-binding targets (common and different between vehicle and A $A$-treated cells) no enrichment is observed (left). Considering unique targets that are only present either in vehicle or A enrichment for FMRP targets in mRNAs differentially bound to polyribosomes in the presence of A. $\mathbf{f}$ Heatmap showing expression changes of FMRP targets between total and ribosomal RNAs unique to $A \beta$, using unsupervised hierarchical clustering. Data represent mean \pm SEM and were analysed using one-way ANOVA with Tukey's correction, or Fisher's exact test. $n=3-5$ biological replicates (individual experiments shown as white dots). ${ }^{*} p<$ $0.05 ;{ }^{* *} p<0.01 ;{ }^{* * *} p<0.001$ 


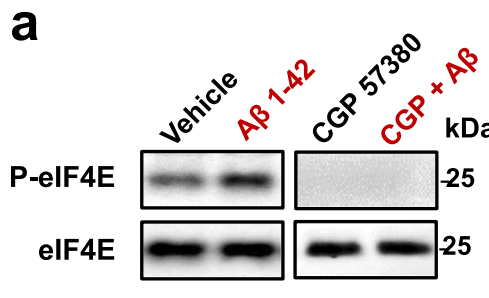

b

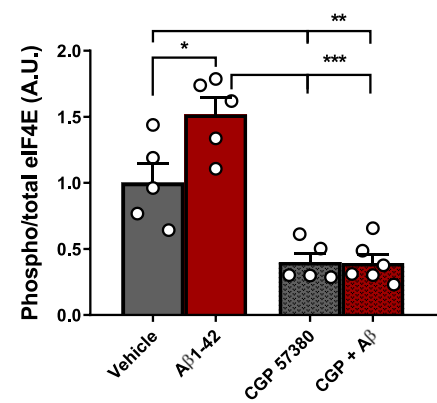

e
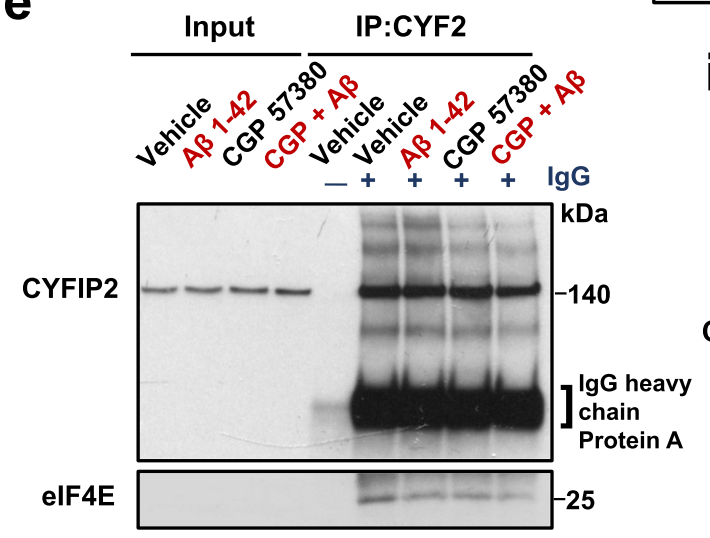

f

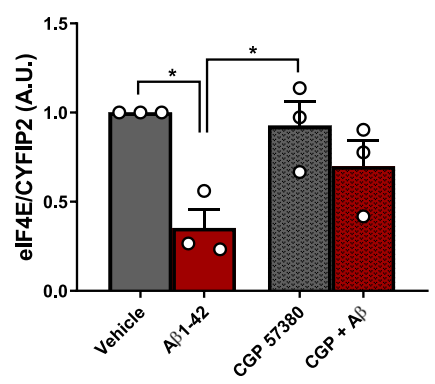

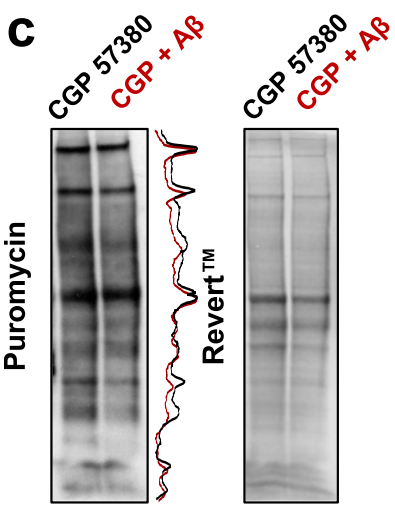
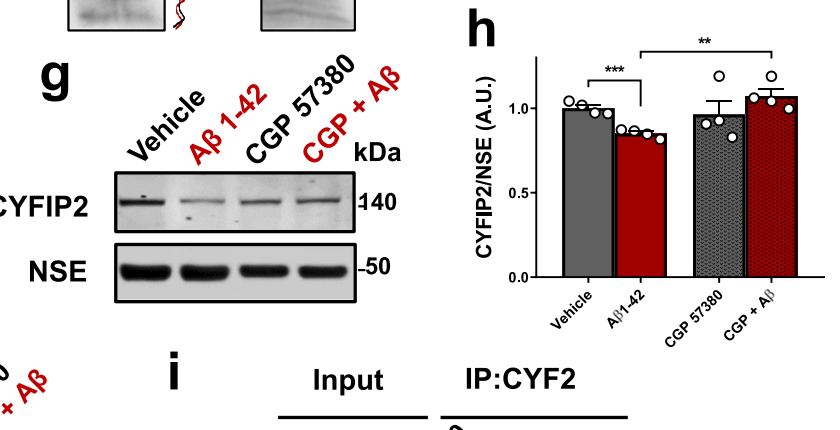

IP:CYF2

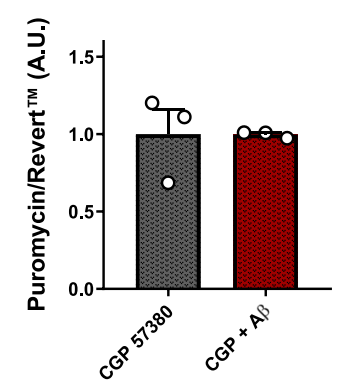

h
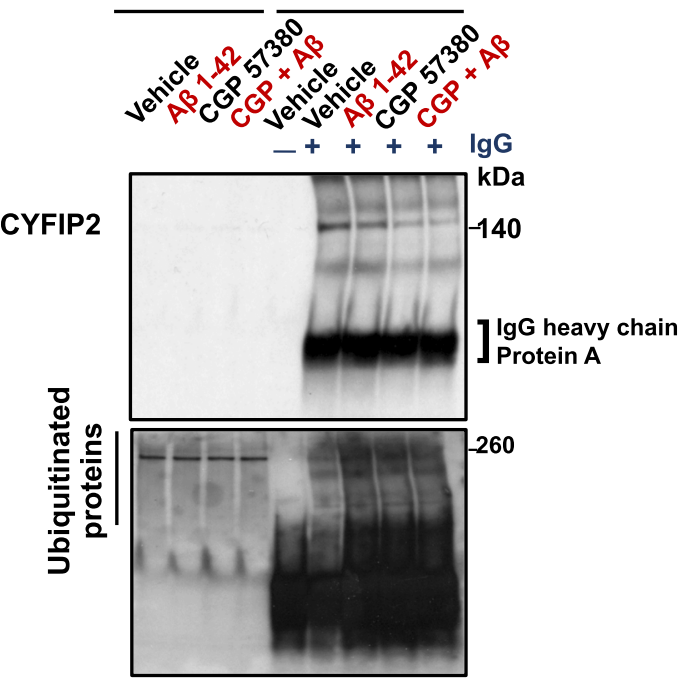

j

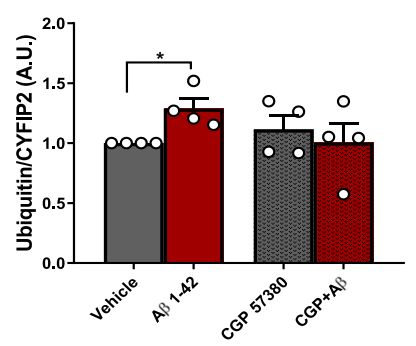

Fig. $2 A \beta_{1-42}$ regulates protein synthesis in neurons via an elF4E-CYFIP2 axis, resulting in CYFIP2 reduction. $\mathbf{a}$, $\mathbf{b} A \beta_{1-42}$ induces increase in elF4E phosphorylation at Ser209 ( $q=4.89, p<0.05)$, and an Mnk1 inhibitor (10 $\mu$ M CGP 57380) reduces this phosphorylation and blocks this effect ( $q$ $=0.06, p>0.99)$. $\mathbf{c}, \mathbf{d}$ CPG 57380 treatments prevent $A \beta_{1-42}$-induced protein synthesis in primary neurons $(t=0.014, p=0.98)$, as determined by SUnSET. The inhibitor does not alter basal protein synthesis ( $t=0.21, p=0.99$, data not shown). e, $\mathbf{f} A \beta_{1-42}$ treatment reduces elF4E binding to CYFIP2, as determined by co-immunoprecipitation ( $q=5.73, p<0.05)$. This effect is prevented by CGP 57380 treatment $(q=2.01, p=0.52)$ which itself does not have an effect $(q=0.66, p=0.96)$. $\mathbf{g}, \mathbf{h}$ A $\beta_{1-42}$ treatment reduces CYFIP2 levels when normalised to levels of neuronal marker NSE $(t=6.92, p<$ $0.001)$, which is prevented by CGP 57380 treatment $(t=1.23, p=0.27) . \mathbf{i}, \mathbf{j}$ A $\beta_{1-42}$ treatment leads to ubiquitination of $C Y F I P 2(t=3.55, p<0.05)$, as determined by co-IP, which is prevented by CGP 57380 treatment $(t=0.56, p=0.60)$ although the inhibitor alone has no effect $(t=1.03, p=0.38)$. Data represent mean \pm SEM and were analysed using one-way ANOVA with Tukey's correction or Student's $t$ test. $n=3-6$ biological replicates 
blocking the eIF4E-eIF4G interaction ${ }^{49}$. As CYFIP2 has an identical eIF4E-binding motif to CYFIP1 $1^{22,24}$, we hypothesised it could also interact with eIF4E. We found that CYFIP2 co-immunoprecipitated with eIF4E, in rat primary cortical neurons at 28 DIV, and in the mouse hippocampus, even in synaptosome-enriched fractions (Supplementary Fig. 4). These data suggest that CYFIP2 interacts with eIF4E. Next, we tested whether $A \beta_{1-42^{-}}$ induced phosphorylation of eIF4E would disrupt the interaction between CYFIP2 and eIF4E, using co-IP experiments in the presence or absence of $A \beta_{1-42}$ (Fig. $2 \mathrm{e}, \mathrm{f})$. We found that treatment with $A \beta_{1-42}$ alone significantly reduced the amount of eIF4E bound to CYFIP2 compared with vehicle-treated cells (Fig. 2e, f). Further, blocking Mnk1 prevented $A \beta_{1-42}$-induced dissociation of eIF4E and CYFIP2 (Fig. 2e, f), and the inhibitor itself did not have any effect (Fig. 2e, f). We also found that $A \beta_{1-42}$ treatment significantly reduced binding of eIF4E to CYFIP1 (Supplementary Fig. 5a, b).

We studied whether the dissociation of eIF4E and CYFIP2 could ultimately lead to reduction of CYFIP2 expression, using immunoblotting to detect CYFIP2 levels in cell lysates which were then normalised to levels of a neuronal marker, neuron specific enolase (NSE). We found that $A \beta_{1-42}$ treatment led to significant CYFIP2 reduction when normalised to NSE (Fig. $2 g$, h), which was prevented by Mnk1 inhibition (Fig. 2g, h). CYFIP1 levels were not changed when normalised to NSE (Supplementary Fig. 5c, d). In addition, the reduction of CYFIP2 expression could not be explained by synapse loss as levels of pre-synaptic marker $\alpha$-synaptotagmin were not altered by $A \beta_{1-42}$ treatment when normalised to NSE levels (Supplementary Fig. 5c, d). Levels of $\alpha$-synaptotagmin were, however, reduced after 5 days of treatment with $100 \mathrm{nM} \mathrm{A} \beta_{1-42}$ (Supplementary Fig. 5e, f), indicating CYFIP2 expression is likely reduced prior to synapse loss.

We hypothesised that the reduction of CYFIP2 may result from its degradation by the proteasome. We therefore stripped the immunoblots for CYFIP2 and CYFIP1 co-IPs and re-probed with an antibody against Ub that recognises ubiquitinated proteins. We detected a significant increase in levels of Ub bound to CYFIP2 in A $\beta_{1-42}$-treated cells compared with vehicle-treated cells (Fig. $2 \mathrm{i}, \mathrm{j}$ ), although the possibility that ubiquitination of proteins interacting with CYFIP2 was also detected cannot be excluded. Inhibition of Mnk1 prevented the effect of $A \beta_{1-42}$ on CYFIP2 ubiquitination (Fig. $2 i, j$ ), and the inhibitor itself did not have any effect (Fig. 2i, j). In contrast, no difference was found in levels of Ub bound to CYFIP1 in $A \beta_{1-42}$-treated cells compared with vehicletreated cells (Supplementary Fig. 5g, h).

To test whether this pathway is genetically associated with $\mathrm{AD}$, we screened variants in five genes, MKNK1, MKNK2, CYFIP2, CYFIP1 and EIF4E, for associations with AD (Supplementary Fig. 6). A total of 1727 variants were identified for the five genes from published summary data $^{35}$. Following the creation of tag SNPs in order to exclude SNPs in high LD, there were 763 selected SNPs within $10 \mathrm{~kb}$ of the coding regions of each gene (Supplementary Table 4). After correcting for multiple testing there were no SNPs associated with AD. The strongest association was observed with SNP rs1258047 $\sim 6.3 \mathrm{~kb}$ downstream of MKNK1; this SNP is an MKNK1-AS1 intronic variant and is also upstream of $K N C N: 2 \mathrm{~kb}$. Mining data from the GTEx project ${ }^{36}$ showed that rs1258047 (or any other variants in $\mathrm{LD}\left(r^{2}>0.8\right)$ ) is not an eQTL for any gene. Taken together, this suggests that no mutations in the chosen pathway associate with $A D$, although more extensive studies will be more conclusive.

\section{CYFIP2 reduction leads to age-dependent accumulation of pre-tangle-like structures in the mouse brain}

Given that CYFIP2 is downregulated in post-mortem $\mathrm{AD}$ brain ${ }^{23}$, in a mouse model of $\mathrm{AD}^{23}$ and in primary cortical neurons following treatment with $A \beta_{1-42}$ oligomers (Fig. 2i, j), we speculated that its reduction may be important in the pathogenesis of AD. Previously, we reported that 3-4-month-old heterozygous Cyfip2 null mutants $\left(C y f i p 2^{+/-}\right)$, having about $50 \%$ reduced CYFIP2 expression, have increased levels of tau phosphorylation at Ser214, possibly due to a post-transcriptional increase in $\alpha$ CaMKII levels ${ }^{23}$. Here, we also found increased tau phosphorylation at Ser416, an $\alpha$ CaMKII-specific phospho-site ${ }^{50,51}$, when normalised to levels of total tau (Supplementary Fig. 7a, b). Further, in the young, adult Cyfip $2^{+/-}$mice no differences were found at pathologyassociated sites of tau phosphorylation AT-8 (Ser202/ Thr205) (Supplementary Fig. 7c, d) or PHF-1 (Ser396/ Ser404) (Supplementary Fig. 7c, d).

However, we hypothesised that priming of tau phosphorylation by $\alpha$ CaMKII in a CYFIP2-dependent manner combined with age-related factors could result in pathological tau phosphorylation at the AT-8 and PHF-1 phosphorylation $\operatorname{sites}^{52}$. We immunohistochemically confirmed previous reports that GSK3 $\beta$ activity dependent on its phosphorylation at Tyr216 is increased with 'healthy' ageing ${ }^{53}$ (Supplementary Fig. 8), which may be a factor in driving pathological tau hyperphosphorylation. This idea was tested with IHC in 12-month-old Cyfip $2^{+/-}$ mice and wild-type littermates, including a group of 3month-old wild-type mice to control for the effects of 'healthy' ageing. In the hippocampus, we observed an increase in neuritic layers across most sub-regions of both AT-8 and PHF-1 immunoreactivity between the 3month-old and 12-month-old wild-type mice (Fig. 3a, b). In 12-month-old Cyfip $2^{+/-}$mice there was a further increase for AT-8 immunoreactivity, specifically in the stratum oriens $(s o)$ and stratum radiatum $(s r)$ of area 


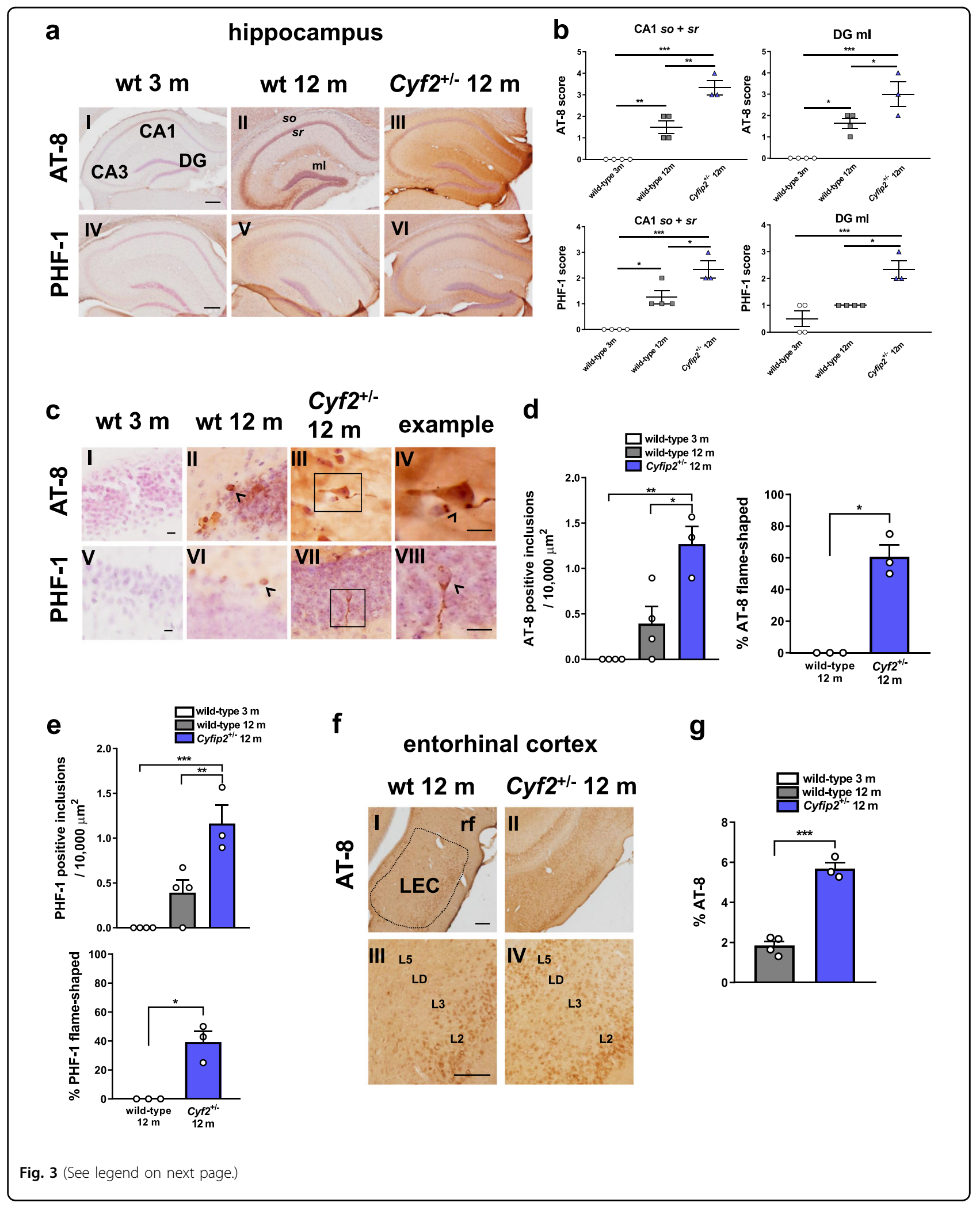


(see figure on previous page)

Fig. 3 Reduced CYFIP2 expression results in phospho-tau accumulation in hippocampal formation and entorhinal cortex of 12-month-old mice. a Representative images of dorsal hippocampal sections from wild-type mice at 3 months and 12 months, and Cyfip $2^{+/-}$mice at 12 months, immunohistochemically stained for AT-8 (I-III) and PHF-1 (IV-VI) phospho-tau and haemotoxylin to label nuclei. Scale bars are $200 \mu \mathrm{m}$. b Analysis of $D A B$ staining in the so and sr of area CA1 and the $\mathrm{ml}$ of the dentate gyrus showing increased phospho-tau with normal ageing (area CA3 not shown) (AT-8: CA1 $q=6.57, p<0.01$, CA3 $q=7.26, p<0.01, \mathrm{DG} q=5.61, p<0.05$; PHF-1: CA1 $q=5.94, p<0.01$, CA3 $q=13.86, p<0.0001$, DG $q=2.19, p=$ 0.32 ) and a further increase in 12-month-old Cyfip2 ${ }^{+/-}$mice specifically in the stratum oriens (so) and stratum radiatum (sr) of area CA1 and in the molecular layer ( $\mathrm{ml}$ ) of the dentate gyrus (DG) (AT-8: CA1 $q=7.44, p<0.01$, DG $q=4.4, p<0.05$; PHF-1: CA1 $q=4.77, p<0.05, \mathrm{DG} q=5.4, p<0.05$ ). No difference was found in area CA3 (AT-8: $q=0.34, p=0.97$; PHF-1: $q=2.14, p=0.34$, data not shown). $\mathbf{c}$ Higher magnification images of hippocampal sections labelled with AT-8 (I-IV) and PHF-1 (V-VIII). d, e Quantification of number of phospho-tau inclusions showing increase in Cyfip2 $^{+/-}$mice (AT-8: $q=5.6, p<0.05$; PHF-1: $q=5.7, p<0.01$ ), but not with healthy ageing (AT-8: $q=2.7, p=0.20 ;$ PHF-1: $q=3.16, p=0.12$ ). Inclusions in Cyfip $2^{+-}$mice had a characteristic flame shape which were absent in aged wild-types (AT-8: $\left.t=8.17, p<0.01 ; \mathrm{PHF}-1: t=5.28, p<0.05\right)$. Scale bars are $10 \mu \mathrm{m}$. f Representative images of AT-8 staining in LEC of 12 -month-old wild-type and Cyfip2 ${ }^{+/-}$mice (I-II). Higher magnification images showing laminar distribution of AT-8 in the LEC (III-IV). $\mathbf{g}$ Quantification of AT-8 positive staining in LEC across all layers showing increase in

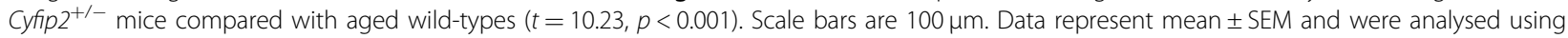
one-way ANOVA with Tukey's correction or Student's $t$ test. $n=3-4$ animals per condition (individual animals shown as white dots). ${ }^{*} p<0.05 ;{ }^{* *} p<$ $0.01 ;{ }^{* * *} p<0.001$. L2-5 layers 2-5, LD lamina dissecans, LEC lateral entorhinal cortex, ml molecular layer, if rhinal fissure, so stratum oriens, sr stratum radiatum.

CA1, and in the molecular layer $(\mathrm{ml})$ of the dentate gyrus (DG) (Fig. 3a, b). No changes were observed in area CA3 (data not shown). Similar results were found for PHF-1 immunoreactivity, with a significant increase in area CA1 and DG (Fig. 3a, b), but no difference in area CA3 (data not shown).

Higher magnification images revealed the presence of more AT-8- and PHF-1-positive inclusions in 12-monthold Cyfip $2^{+/-}$mice in comparison to wild-type littermates (Fig. 3c-e). In contrast, 12-month-old wild-type mice did not have significantly more inclusions than 3-month-old wild-type mice (Fig. 3c-e). Importantly, among the inclusions in the Cyfip $2^{+-}$hippocampus, several had a flame shape characteristic of pre-tangles and NFTs, which were never found in the wild-type littermates (Fig. 3c-e). These are likely to be pre-tangles due to the lack of positive staining in the hippocampus with Amytracker ${ }^{\mathrm{TM}}$, which, like Thioflavins, detects cross- $\beta$-sheet structures including NFTs ${ }^{54}$ (see next section; Supplementary Fig. 12a, IV-VI).

AT-8 and PHF-1 immunoreactivity was increased in hippocampal area CA1 and DG, but not in area CA3, of 12-month-old Cyfip $2^{+/-}$mice (Fig. 3a, b). These two subregions receive direct inputs from the entorhinal cortex where tau pathology is believed to initiate ${ }^{55,56}$. Therefore, we examined AT-8 and PHF-1 immunoreactivity in the lateral entorhinal cortex (LEC) of 12-month-old wild-type and Cyfip $2^{+/-}$mice. AT-8 immunoreactivity was significantly increased in LEC of 12-month-old Cyfip $2^{+l-}$ mice compared with wild-type littermates (Fig. 3f, g). A qualitative comparison of the laminar distribution of AT8 revealed that in wild-type mice, the staining was restricted to layers 2 and 3, as has been described previously in 'healthy' ageing studies ${ }^{57}$, whereas in Cyfip $2^{+/-}$ mice, AT-8 immunoreactivity had progressed into deeper layers, including layer 5 (Fig. 3f, III-IV). No differences were found for PHF-1 IHC in LEC of 12-month-old wildtype or Cyfip $2^{+/-}$mice (Supplementary Fig. 9a, b).

To test whether increased AT-8 and PHF-1 immunoreactivity in the hippocampus could be due to augmented levels of tau protein, which itself is subject to local synthesis $^{58,59}$, we assessed tau immunostaining in mouse brain sections. No differences were found in dendritic layers for CA1 or DG between 3-month-old and 12month-old wild-type mice (Supplementary Fig. 10a-c). A significant increase was found in $s o$ and $s r$ of area CA1 of 12-month-old Cyfip $2^{+/-}$mice compared with agematched wild-types, but no difference was found in the $\mathrm{ml}$ of the DG (Supplementary Fig. 10a, c). Since the change in tau expression is much smaller, or even not detected, in comparison to the observed upregulation in tau phosphorylation at the AT-8 and PHF-1 sites (Fig. 3a, b), it is unlikely that the changes in tau phosphorylation are simply due to altered levels of tau protein expression. The differences observed were not due to background staining or age-related build-up of proteins (Supplementary Fig. 11).

\section{CYFIP2 reduction results in $A \beta$-positive accumulations in the mouse thalamus and astrocytic and microglial responses in the mouse hippocampus}

Previously, we reported that 3-4-month-old Cyfip $2^{+/-}$ mice had higher levels of soluble oligomeric $A \beta_{1-42}$ in the hippocampus compared with age-matched wild-type mice $^{23}$. We therefore hypothesised that increased levels of oligomeric $A \beta_{1-42}$ could aggregate with age to result in $\mathrm{A} \beta_{1-42}$ accumulation in older mice, as it has been reported that mouse $A \beta_{1-42}$ can aggregate, although less efficiently than human $A \beta_{1-42}{ }^{41}$. We used immunohistochemical methods to search for amyloid plaques in 12-month-old wild-type and Cyfip $2^{+/-}$mice, using 3-month-old wildtype mice as control for effects of healthy ageing. 
We used an anti-A $\beta$ 4G8 antibody (aa 17-24) to detect endogenous rodent $A \beta_{1-42}$ in mouse brain sections. In the hippocampus no positive immunohistochemical staining was observed for 4G8 across the three groups (Supplementary Fig. 12a I-III). However, in the thalamus, although no differences were observed for 4G8-positive staining between 3-month-old and 12-month-old wildtype mice (Fig. 4a I and II, b), a significant increase was found in 12-month-old Cyfip $2^{+/-}$mice when compared with age-matched controls (Fig. 4a II and III, b). This was reflected by the observation of several plaque-like inclusions localised predominantly to the somatosensory nuclei of the thalamus in these mice (Fig. 4a, IV).

To further confirm the identity of these inclusions we also used the Amytracker ${ }^{\mathrm{TM}}$ range of fluorescent tracer molecules on brain sections. These dyes function in a manner similar to Thioflavins, cell-permeable benzothiazole dyes that fluoresce upon binding to cross- $\beta$-sheet structures, a defining feature of amyloid fibrils, and are therefore typically used for detection of amyloid plaques $^{54}$. Amytracker ${ }^{\mathrm{TM}}$ allows improved amyloid detection compared with Thioflavin compounds, detecting levels of both fibrillar and pre-fibrillar proteins and peptides ${ }^{54}$. The dye revealed a trend for an increase in staining between 3month-old and 12-month-old wild-type hippocampus (Supplementary Fig. 12a IV and V, b), but no further change was observed in 12-month-old Cyfip $2^{+/-}$mice (Supplementary Fig. 12a VI, b). However, in the thalamus, Amytracker ${ }^{680}$ showed significantly increased staining in 12-month-old wild-type mice compared with 3-monthold wild-types (Fig. 4a V and VI, c). This suggests that the dye most likely recognises other amyloid-like proteins besides $A \beta$, which are increased with ageing ${ }^{60}$. A further significant increase was found in 12-month-old Cyfip $2^{+/-}$ mice (Fig. 4a VII, c). The dye also detected thalamic inclusions that resembled amyloid plaques, which were not present in age-matched wild-type mice (Fig.4a, VIII). These data strongly suggest that $A \beta_{1-42}$ can accumulate in an age-dependent manner upon CYFIP2 reduction, although further characterisation of these inclusions is required.

Although plaques and tangles are two of the characteristic hallmarks of $\mathrm{AD}$, there are several other pathological features such as gliosis, which is thought to be reactive to amyloid and tau pathology. To test whether 12-month-old Cyfip $2^{+/-}$mice have an astroglial response, mouse brain sections were analysed for glial fibrillary acidic protein (GFAP) expression, using IF. Increased numbers of GFAP-positive astrocytes were found across all hippocampal sub-regions except the hilus in 12month-old wild-type mice compared with 3-month-old wild-types; a further significant increase was observed in 12-month-old Cyfip2 $2^{+-}$mice compared with agematched wild-types, specifically in area CA1 and DG, but not area CA3 or hilus (Fig. 4a, b). To test for the presence of a microglial response in 12-month-old Cyfip $2^{+/-}$mice, we used an antibody against ionised calcium-binding adapter molecule 1 (Iba1), a microglia/ macrophage-specific calcium-binding protein, to ubiquitously detect microglia in mouse brain sections by IF. We found a significant increase in the number of Iba1-positive microglia in the CA1 region of 12-month-old wild-type mice compared with 3-month-old wild-type mice, and a trend for an increase in area CA3 and DG (Fig. 4c, d). In 12-month-old Cyfip $2^{+/-}$mice we found a further increase in area CA1 and hilus compared with age-matched controls (Fig. 4c, d). Taken together, our data show that Cyfip $2^{+/-}$mice display hippocampal gliosis.

Given that $A \beta$ accumulations were found in the thalamus of aged Cyfip $2^{+/-}$mice and glial responses have been reported near the vicinity of plaques ${ }^{61,62}$, astrocytes and microglia were also quantified in the thalamus of mouse brain sections. Increased numbers of GFAP-positive astrocytes (Supplementary Fig. 13a I-III, b) and Iba1positive microglia (Supplementary Fig. 13a IV-VI, c) were found in 12-month-old wild-type mice compared with 3month-old wild-type mice. No further changes in astrocyte and microglial numbers (Supplementary Fig. 13a-c) were observed in the thalamus of 12-month-old Cyfip $2^{+/-}$ mice. These data may suggest that the glial response in Cyfip $2^{+/-}$mice is reactive to phospho-tau or soluble $A \beta_{1-42}{ }^{23}$, rather than $A \beta$ accumulation.

\section{CYFIP2 reduction leads to dendritic spine loss in hippocampal CA1 neurons and impacts on contextual fear memory formation}

Previously, we reported a change in dendritic spine morphology on apical dendrites of hippocampal CA1 neurons in 3-4-month-old Cyfip $2^{+/-}$mice compared with age-matched wild-type mice ${ }^{23}$. Specifically, young, adult Cyfip $2^{+/-}$mice have more immature long/thin-type spines and fewer mature stubby/mushroom-type spines, but the overall spine number is not changed ${ }^{23}$. Since with ageing CYFIP2 reduction causes tau pathology and gliosis in the hippocampus (Figs. 3 and 4), we tested whether this may result in spine loss in area CA1. We used Golgi-Cox impregnation to label neurons in brains from 12-monthold wild-type and Cyfip $2^{+/-}$mice, and quantified dendritic spines in the stratum radiatum of hippocampal CA1. We found that 12-month-old Cyfip $2^{+/-}$mice had an overall reduction in the number of dendritic spines compared with age-matched wild-types (Fig. 5a, b). Upon stratification, there was a trend for reduction in the number of stubby spines and a significant reduction in the number of mushroom spines, but no difference in thin spines or filopodia (Fig. 5c).

The age-related spine loss in Cyfip $2^{+/-}$mice is expected to exacerbate learning and memory deficits. We tested 


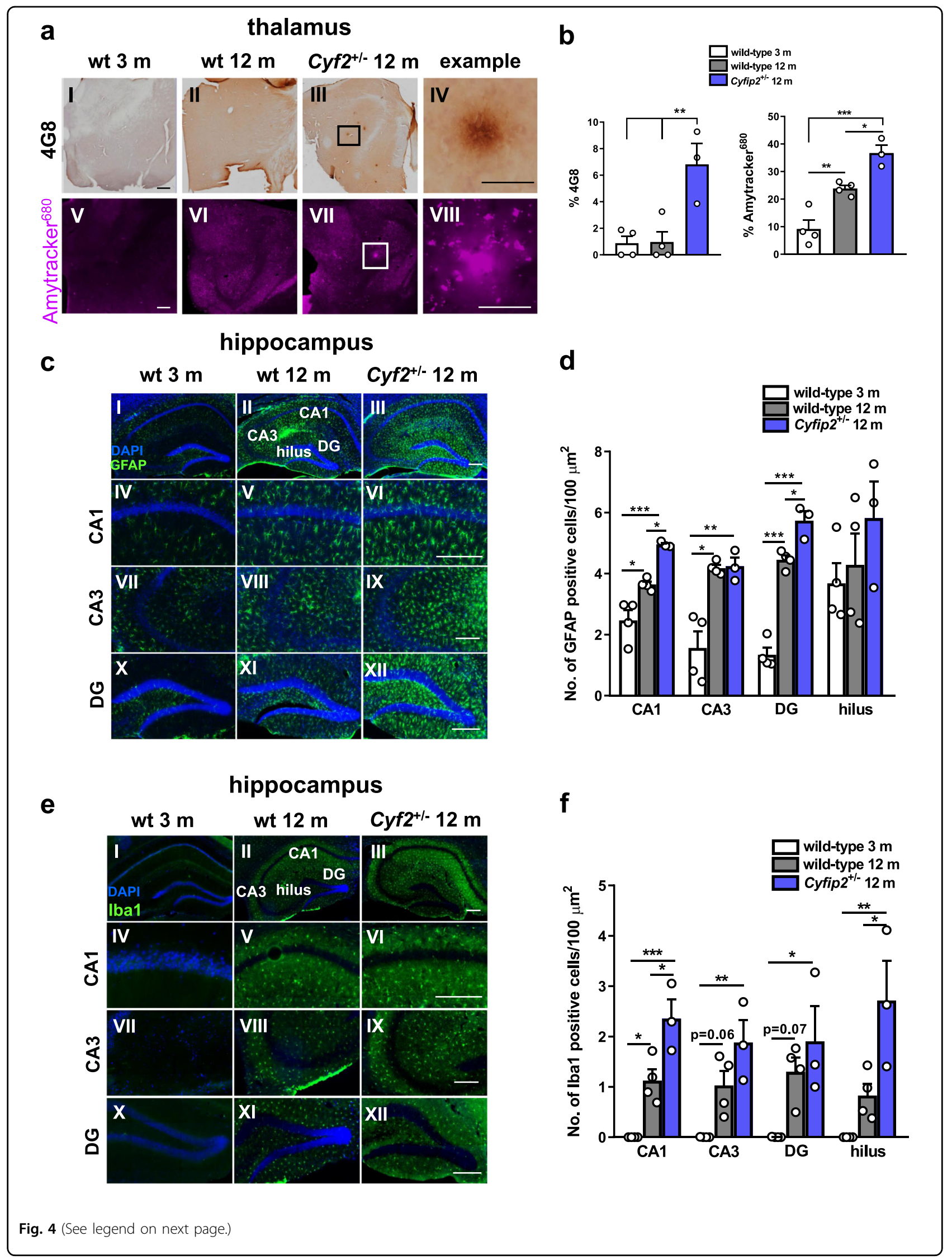


(see figure on previous page)

Fig. 4 Reduced CYFIP2 expression results in thalamic $A \beta$ accumulation and astrocytic and microglial responses in specific hippocampal sub-regions of 12-month-old mice. a Representative images of the thalamus from dorsal brain sections of wild-type mice at 3 months and 12 months, and Cyfip2 $2^{+-}$mice at 12 months, labelled with an anti-4G8 antibody to detect A $\beta$ (I-IV) and Amytracker ${ }^{680}$ (V-VIII). Scale bars are $50 \mu \mathrm{m}$ in 'example' panels and $500 \mu \mathrm{m}$ in all other panels. b Quantification of $4 \mathrm{G} 8$ staining, expressed as \% of area sampled, detects $A \beta$ accumulation in Cyfip $2^{+/}$mice compared with aged wild-types $(q=6.07, p<0.01)$, and no difference between young adult and aged wild-type mice $(q=0.11, p=$ 0.99). Quantification of Amytracker ${ }^{680}$ staining, expressed as $\%$ of area sampled, showed increased staining with healthy ageing $(q=6.04, p<0.01)$, and a further significant increase in Cyfip $2^{+/-}$mice $(q=4.92, p<0.05)$. c Representative images of dorsal hippocampal sections (I-III) from wild-type mice at 3 months and 12 months, and Cyfip2 ${ }^{+/-}$mice at 12 months, stained for GFAP. Higher magnification images of CA1 (IV-VI), CA3 (VII-IX) and DG (X-XII). Scale bars are $200 \mu \mathrm{m}$. d Quantification of GFAP-positive astrocytes in hippocampal sub-regions shown indicates an elevated astrocytic response across all hippocampal sub-regions except the hilus in 12-month-old wild-type mice compared with 3-month-old wild-types (CA1 $q=5.42$, $p<0.05$, CA3 $q=7.25, p<0.01$, DG $q=14.49, p<0.0001$, hilus $q=0.67, p=0.88$ ), and a further significant increase in 12-month-old Cyfip $2^{+/-}$mice compared with age-matched wild-types, specifically in area CA1 and DG, but not area CA3 or hilus (CA1 $q=5.59, p<0.05, \mathrm{CA} 3 q=0.16, p=0.99, \mathrm{DG}$ $q=5.54, p<0.05$, hilus $q=1.56, p=0.54$ ). e Representative images of dorsal hippocampal sections (I-III) from wild-type mice at 3 months and 12 months, and Cyfip2 $2^{+-}$mice at 12 months, stained for Iba1. Higher magnification images of CA1 (IV-VI), CA3 (VII-IX) and DG (X-XII). Scale bars are $200 \mu \mathrm{m}$. f Quantification of Iba1-positive microglia in hippocampal sub-regions shown reveals an increased microglial response in area CA1 of 12month-old wild-type mice compared with 3-month-old wild-type mice, and a trend for an increase in area CA3 and DG (CA1 $q=5.22, p<0.05$, CA3 $q=3.9, p=0.06, \mathrm{DG} q=3.74, p=0.07$, hilus $q=2.25, p=0.30$ ) and a further increase in 12-month-old Cyfip ${ }^{+/-}$mice compared with age-matched wild-types, specifically in area CA1 and hilus (CA1 $q=5.31, p<0.05$, CA3 $q=3.01, p=0.15$, DG $q=1.62, p=0.51$, hilus $q=4.74, p<0.05$ ). Data represent mean \pm SEM and were analysed using one-way ANOVA with Tukey's correction. $n=3-4$ animals per condition (individual animals shown as white dots). ${ }^{*} p<0.05 ;{ }^{* *} p<0.01 ;{ }^{* *} p<0.001$.

this in hippocampus-dependent contextual fear conditioning, using Cyfip $2^{+/-}$mice and wild-type littermates at 3-4 months or 12 months of age. We found that $12-$ month-old Cyfip $2^{+/-}$mice froze significantly less than 3-4-month-old Cyfip $2^{+/-}$mice and 12-month-old wildtype mice (Fig. 5d) strongly suggesting that ageing exacerbates memory impairment in Cyfip $2^{+/-}$mice.

\section{Discussion}

The loss of synapses in $\mathrm{AD}$ is the best correlate of memory impairment and may result from build-up of amyloid-beta $\left(\mathrm{A} \beta_{1-42}\right)$ peptides during disease progression. However, the precise roles of $A \beta_{1-42}$ in synapse modification are poorly understood. Here, we show that in neurons $A \beta_{1-42}$ regulates mRNA translation and modulates ribosomal association of several FMRP-bound mRNAs, primarily affecting synthesis of proteins related to synaptic function. We demonstrate that this is likely to be via a pathway involving Mnk1 and eIF4E, which results in reduction of the 4E-binding protein CYFIP2, most likely due to ubiquitination. Finally, we find that reduced expression of CYFIP2 in 12-month-old mice causes several phenotypes of the disease. These include development of phospho-tau accumulation in the hippocampus and entorhinal cortex, thalamic $A \beta$ accumulation, hippocampal gliosis, dendritic spine loss and impaired hippocampus-dependent memory formation.

We propose a model where CYFIP2 reduction functions as both a cause and consequence of $\mathrm{AD}$ pathogenesis (Fig. 5e). Reduction of CYFIP2 levels may result from activation of the Mnk1-eIF4E-CYFIP axis by $\mathrm{A} \beta_{1-42}$ (Fig. 2). Once lost, a state of prolonged protein synthesis is proposed to further contribute to the toxic loop of $A \beta_{1-42}$ production and tau hyperphosphorylation via increased synthesis of APP and $\alpha$ CaMKII protein, respectively ${ }^{23}$. Ageing processes, such as Tyr216 phosphorylationdependent activity of GSK $3 \beta^{53}$, may then interact to drive accumulation of $A \beta_{1-42}$ and hyperphosphorylated tau (Figs. 3 and 4 ) in the form of plaques and tangles. With age, CYFIP2 reduction also promotes a glial response (Fig. 4), which is likely to be secondary to neuronal injury due to the absence of CYFIP2 expression in glial cells ${ }^{23}$. Functionally, CYFIP2 reduction may cause a failure of immature spines to stabilise into mushroom spines via reduced WAVE function ${ }^{63}$, resulting in agedependent loss of dendritic spines (Fig. 5). This correlates with an initial spatial memory retention deficit ${ }^{23}$, which is then further exacerbated to an additional impairment in contextual memory formation with age (Fig. 5).

The presence of key AD-like characteristics in 12-monthold Cyfip $2^{+/-}$mice suggests it is useful as a model of AD. Amyloid pathology has previously been detected upon overexpression of mutant human APP, or humanisation of the murine $A \beta_{1-42}$ sequence in a knock-in model ${ }^{64}$. Rodent $A \beta_{1-42}$ differs from the human sequence by three amino acids $\left(\mathrm{Arg}^{5}, \mathrm{Tyr}^{10}\right.$, and $\mathrm{His}^{13}$ are replaced by Gly, Phe and Arg, respectively); as $\mathrm{His}^{13}$ is required for $A \beta_{1-42}$ fibrillogenesis, it makes rodent $A \beta_{1-42}$ less prone to forming amyloid aggregates ${ }^{39}$. A proteolytic processing difference between species may result in protection of the mouse sequence against $\beta$-secretase processing ${ }^{40}$; however, overexpression of murine $A \beta_{1-42}$ can lead to formation of morphologically distinct plaques in an age-dependent manner, suggesting rodent $A \beta_{1-42}$ can aggregate, but less efficiently than the human peptide ${ }^{41}$. At 12 months, Cyfip $2^{+-}$mice develop $A \beta_{1-42}$ accumulations in the thalamus, a vulnerable brain region ${ }^{65,66}$, which is understudied in $\mathrm{AD}$ research ${ }^{67}$. Further characterisation will therefore be 


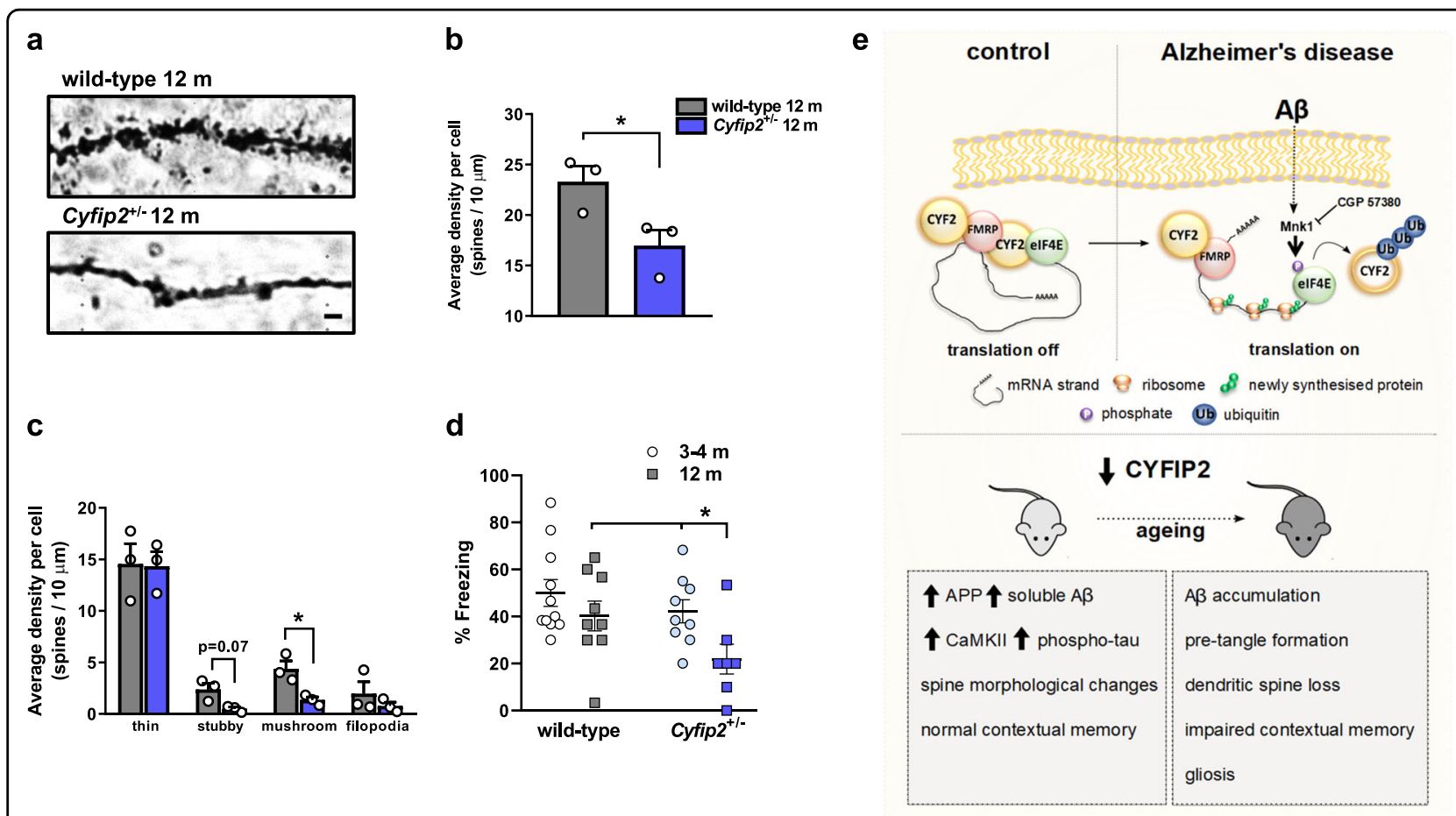

Fig. 5 Reduced CYFIP2 expression causes loss of dendritic spines and age-dependent impairment in contextual memory formation. a Representative images of apical dendrites from hippocampal CA1 neurons impregnated with Golgi-Cox stain, from 12-month-old wild-type and

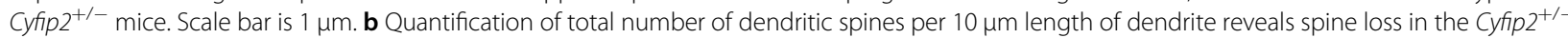
mice $(t=2.84, p<0.05)$. c Quantification of numbers of dendritic spines stratified according to morphological parameters, indicating loss of mushroom $(t=3.76, p<0.05)$ and stubby spines $(t=3.04, p=0.07)$ in Cyfip $2^{+/-}$mice, but no difference in numbers of thin spines $(t=0.09, p=0.93)$ or filopodia ( $t=0.98, p=0.42$ ). $\mathbf{d}$ Quantification of percentage freezing in 3-4-month-old and 12-month-old wild-type and Cyfip $2^{+/-}$mice in the contextual fear-conditioning paradigm, showing significant differences in genotype and age for freezing (effect of genotype $F_{1,32}=4.84, p<0.05$; effect of age $F_{1,32}=6.47, p<0.05$; genotype and age interaction $F_{1,32}=0.78, p=0.38$ ). Post-hoc analysis shows 12 -month-old Cyfip2 ${ }^{+/-}$mice freeze significantly less than 3-4-month-old Cyfip $2^{+/-}$mice $(q=3.25, p<0.05)$ and 12-month-old wild-type mice $(q=2.92, p<0.05)$. Data represent mean \pm SEM and were analysed using Student's $t$ test or two-way ANOVA with Student-Newman-Keuls correction. $n=3$ animals per condition for spine analysis, $n=7-11$ animals per condition for behaviour (individual animals shown as dots or squares). ${ }^{*} p<0.05$. e Proposed model of CYFIP2 dysregulation as a cause and consequence in Alzheimer's disease pathogenesis. The schematic in the upper panel shows that in neurons of the healthy brain, mRNAs bound by FMRP are translationally repressed via the elF4E-binding protein CYFIP2. In Alzheimer's disease, increased production and/or reduced clearance of $A \beta$ results in dissociation of this complex and prolonged translation of a subset of these mRNAs due to reduction of CYFIP2, which can be blocked by inhibition of Mnk1. The lower panel shows that in the young-adult mouse brain, loss of CYFIP2 elevates A $\beta$ production and tau phosphorylation (at CaMKIl sites), causes changes in dendritic spine morphology, and impairs spatial, but not contextual, memory. These effects are exacerbated by ageing, leading to accumulation of A $\beta$ and of tau hyperphosphorylated at AT- 8 and PHF- 1 sites, dendritic spine loss, contextual memory impairment, and gliosis.

required of these $\mathrm{A} \beta_{1-42}$-positive inclusions in the Cyfip $2^{+/-}$ mouse model.

AD-related tau pathology is particularly difficult to model, generally only occurring independently when nonphysiological amounts of tau protein are overexpressed or when a point mutant version of tau is expressed, with the limitation that MAPT mutations do not cause $\mathrm{AD}^{64}$. Similarly, pre-tangle-like tau inclusions are only found following co-expression of familial AD-causing mutant human $\mathrm{APP}^{64}$, and not in double knock-in mice having both humanised APP and tau ${ }^{68}$. Instead, the Cyfip $2^{+/-}$ mouse model, which lacks one copy of an endogenous gene, rendering lower expression of CYFIP2 similar as that present in post-mortem $\mathrm{AD}$ brain $^{23}$, shows stereotypical progression of phospho-tau accumulation $^{55,69}$, and may therefore be a more physiological model of sporadic AD-related tau pathology. This has the promise to allow mechanistic understanding of the emergence of both amyloid and tau abnormalities in AD.

Our study shows direct evidence that nanomolar concentrations of $A \beta_{1-42}$ preparations that include oligomers increase mRNA translation, particularly of specific transcripts that are predicted to be regulated by FMRP. The presence of unique candidates within both vehicle- and $A \beta_{1-42}$-treated groups suggests the regulation of translation by $\mathrm{A} \beta_{1-42}$ is complex, as some mRNAs are preferentially translated or untranslated, while many mRNA transcripts are unaffected. Further analysis will be 
required to identify common elements of these transcripts, as well as association or localisation to distinct polyribosomal populations. Our data suggest evidence for a biphasic regulation of protein synthesis in AD. In earlier stages there could be an increase in translation of specific mRNA transcripts, eventually leading to deposition of particular proteins. In later stages of the disease global protein synthesis may be shunted as a compensatory respons $^{70}$, ultimately leading to neurodegeneration ${ }^{71}$. Several lines of evidence suggest that prior to inducing neurotoxicity $A \beta_{1-42}$ can function as a beneficial agent ${ }^{72}$, with its functions ranging from anti-microbial to enhancement of long-term potentiation and memory, akin to a neurotrophic factor ${ }^{73}$. Our finding that nanomolar doses of $A \beta_{1-42}$ enhance translation overall, via a pathway also modulated by the neurotrophin $\mathrm{BDNF}^{74}$, lends support to this idea. As $A \beta_{1-42}$-induced signalling results in dissociation between eIF4E and both CYFIP1 and CYFIP2, it might regulate the translation of both CYFIP1 and 2-regulated mRNAs. However, the reduction of CYFIP2, but not CYFIP1, may act as a switch between reversible and irreversible processes, driving pathology.

Given that our data suggest that CYFIP2 reduction by $A \beta_{1-42}$ can be blocked by Mnk1 inhibition, and CYFIP2 reduction results in age-dependent AD-like pathology in the mouse brain, we propose Mnk1 as a therapeutic target in AD. Our study finds tentative evidence that Mnk1 dysregulation may be associated with $\mathrm{AD}$, and a previous report indicates excessive eIF4E phosphorylation in the $\mathrm{AD}$ brain $^{43}$. The usefulness of Mnk inhibitors for the treatment of $\mathrm{AD}$ has already been noted, in part due to the role of these kinases in tau phosphorylation (patent no. WO2009065596A2) ${ }^{75}$. Our study sheds further light on the involvement of Mnk1 in AD, highlighting novel therapeutic avenues. The compound used in this study is unstable in vivo; however, a different Mnk inhibitor such as eFT508 which is already in clinical trials for treatment of solid tumours and lymphomas ${ }^{45}$ may be suitable.

In conclusion, our study suggests that CYFIP2 reduction plays a pivotal role in $\mathrm{AD}$ pathogenesis and modelling its dysregulation will provide more physiological models of the disease. Our data also identify an upstream pathway which may be implicated in disease pathogenesis and can therefore be targeted for therapeutic intervention.

\footnotetext{
Acknowledgements

We thank Kei Cho, Wendy Noble, and Alessio Delogu for comments on the paper. We also thank Peter Davies for kindly providing us with PHF-1 antibody, Wendy Noble for Ibal antibody and Amytracker ${ }^{\mathrm{TM}}$ dye, and Claire Troakes for 4G8 antibody. This work was supported by an MRC IOPPN excellence Ph.D. studentship and an Alzheimer's Research UK (ARUK) King's College London Network Centre pump priming grant to A.G., and an ARUK pilot grant (ARUKPPG2018A-002) to K.P.G. The use of the Nikon Eclipse Ti2 inverted microscope was possible due to funding by ARUK-EG2013B-1 and the Nikon Wohl Cellular Imaging Centre (WCIC).
}

\section{Author contributions}

A.G. and K.P.G. designed the project and wrote the paper. A.G. performed most of the experiments and analysed the data. R.T.M.N. advised on the polyribosome experiment, analysed the RNA sequencing data, and wrote the relevant sections. K.M. and S.S.T. performed behavioural experiments and K.M. wrote the relevant sections. P.P. performed the GWAS analysis and wrote the relevant sections. B.G.P.N. performed the Native PAGE. E.G. provided all the primary neurons used in this study. All authors discussed the results and commented on the paper.

\section{Conflict of interest}

The authors declare that they have no conflict of interest.

\section{Publisher's note}

Springer Nature remains neutral with regard to jurisdictional claims in published maps and institutional affiliations.

Supplementary Information accompanies this paper at (https://doi.org/ 10.1038/s41398-020-00882-7).

Received: 27 February 2020 Revised: 4 May 2020 Accepted: 7 May 2020 Published online: 16 June 2020

\section{References}

1. Glenner, G. G. \& Wong, C. W. Alzheimer's disease: initial report of the purification and characterization of a novel cerebrovascular amyloid protein. Biochem. Biophys. Res. Commun. 120, 885-890 (1984).

2. Kosik, K. S., Joachim, C. L. \& Selkoe, D. J. Microtubule-associated protein tau (tau) is a major antigenic component of paired helical filaments in Alzheimer disease. Proc. Natl. Acad. Sci. USA 83, 4044-4048 (1986).

3. Alzheimer, A., Stelzmann, R. A., Schnitzlein, H. N. \& Murtagh, F. R. An English translation of Alzheimer's 1907 paper, "Uber eine eigenartige Erkankung der Hirnrinde". Clin. Anat. 8, 429-431 (1995).

4. Goedert, M. Oskar Fischer and the study of dementia. Brain 132, 1102-1111 (2009).

5. DeKosky, S. T. \& Scheff, S. W. Synapse loss in frontal cortex biopsies in Alzheimer's disease: correlation with cognitive severity. Ann. Neurol. 27, 457-464 (1990).

6. Lambert, M. P. et al. Diffusible, nonfibrillar ligands derived from Abeta1-42 are potent central nervous system neurotoxins. Proc. Natl. Acad. Sci. USA 95, 6448-6453 (1998)

7. Walsh, D. M. et al. Naturally secreted oligomers of amyloid beta protein potently inhibit hippocampal long-term potentiation in vivo. Nature 416, 535-539 (2002).

8. Muller, U. C., Deller, T. \& Korte, M. Not just amyloid: physiological functions of the amyloid precursor protein family. Nat. Rev. Neurosci. 18, 281-298 (2017).

9. Westmark, C. J. \& Malter, J. S. FMRP mediates mGluR5-dependent translation of amyloid precursor protein. PLoS Biol. 5, e52 (2007).

10. Westmark, C. J. What's hAPPening at synapses? The role of amyloid betaprotein precursor and beta-amyloid in neurological disorders. Mol. Psychiatry 18, 425-434 (2013)

11. Darnell, J. C. et al. FMRP stalls ribosomal translocation on mRNAs linked to synaptic function and autism. Cell 146, 247-261 (2011).

12. Ascano, M. Jr. et al. FMRP targets distinct mRNA sequence elements to regulate protein expression. Nature 492, 382-386 (2012).

13. Maurin, T. et al. HITS-CLIP in various brain areas reveals new targets and new modalities of RNA binding by fragile $X$ mental retardation protein. Nucleic Acids Res. 46, 6344-6355 (2018).

14. $\mathrm{Li}, \mathrm{Z}$. et al. The fragile $X$ mental retardation protein inhibits translation via interacting with mRNA. Nucleic Acids Res. 29, 2276-2283 (2001).

15. Richter, J. D., Bassell, G. J. \& Klann, E. Dysregulation and restoration of translational homeostasis in fragile $X$ syndrome. Nat. Rev. Neurosci. 16, 595-605 (2015).

16. Dictenberg, J. B., Swanger, S. A., Antar, L. N., Singer, R. H. \& Bassell, G. J. A direct role for FMRP in activity-dependent dendritic mRNA transport links filopodial-spine morphogenesis to fragile $X$ syndrome. Dev. Cell. 14, 926-939 (2008). 
17. Feng, $Y$. et al. Fragile $X$ mental retardation protein: nucleocytoplasmic shuttling and association with somatodendritic ribosomes. J. Neurosci. 17, 1539-1547 (1997).

18. Schenck, A., Bardoni, B., Moro, A., Bagni, C. \& Mandel, J. L. A highly conserved protein family interacting with the fragile $X$ mental retardation protein (FMRP) and displaying selective interactions with FMRP-related proteins FXR1P and FXR2P. Proc. Natl. Acad. Sci. USA 98, 8844-8849 (2001).

19. Kumar, $V$. et al. C57BL/6N mutation in cytoplasmic FMRP interacting protein 2 regulates cocaine response. Science342, 1508-1512 (2013).

20. Pathania, M. et al. The autism and schizophrenia associated gene CYFIP1 is critical for the maintenance of dendritic complexity and the stabilization of mature spines. Transl. Psychiatry 4, e374 (2014).

21. Davenport, E. C. et al. Autism and schizophrenia-associated CYFIP1 regulates the balance of synaptic excitation and inhibition. Cell Rep. 26, 2037-2051 e2036 (2019).

22. Napoli, I. et al. The fragile $X$ syndrome protein represses activity-dependent translation through CYFIP1, a new 4E-BP. Cel/134, 1042-1054 (2008).

23. Tiwari, S. S. et al. Alzheimer-related decrease in CYFIP2 links amyloid production to tau hyperphosphorylation and memory loss. Brain 139, 2751-2765 (2016).

24. Marcotrigiano, J., Gingras, A. C., Sonenberg, N. \& Burley, S. K. Cap-dependent translation initiation in eukaryotes is regulated by a molecular mimic of elF4G. Mol. Cell. 3, 707-716 (1999).

25. Cory, G. O. \& Ridley, A. J. Cell motility: braking WAVEs. Nature 418, 732-733 (2002).

26. Eden, S., Rohatgi, R., Podtelejnikov, A. V., Mann, M. \& Kirschner, M. W. Mechanism of regulation of WAVE1-induced actin nucleation by Rac1 and Nck. Nature 418, 790-793 (2002).

27. Chen, $Z$. et al. Structure and control of the actin regulatory WAVE complex. Nature 468, 533-538 (2010).

28. De Rubeis, S. et al. CYFIP1 coordinates mRNA translation and cytoskeleton remodeling to ensure proper dendritic spine formation. Neuron 79, 1169-1182 (2013).

29. Skarnes, W. C. et al. A conditional knockout resource for the genome-wide study of mouse gene function. Nature 474, 337-342 (2011).

30. Harris, K. M., Jensen, F. E. \& Tsao, B. Three-dimensional structure of dendritic spines and synapses in rat hippocampus (CA1) at postnatal day 15 and adult ages: implications for the maturation of synaptic physiology and long-term potentiation. J. Neurosci. 12, 2685-2705 (1992).

31. Bell, K. A., O'Riordan, K. J., Sweatt, J. D. \& Dineley, K. T. MAPK recruitment by beta-amyloid in organotypic hippocampal slice cultures depends on physical state and exposure time. J. Neurochem. 91, 349-361 (2004).

32. Schmidt, E. K., Clavarino, G., Ceppi, M. \& Pierre, P. SUnSET, a nonradioactive method to monitor protein synthesis. Nat. Methods 6, 275-277 (2009).

33. Trapnell, C. et al. Transcript assembly and quantification by RNA-Seq reveals unannotated transcripts and isoform switching during cell differentiation. Nat. Biotechnol. 28, 511-515 (2010).

34. Anders, S. \& Huber, W. Differential expression analysis for sequence count data. Genome Biol. 11, R106 (2010).

35. Kunkle, B. W. et al. Genetic meta-analysis of diagnosed Alzheimer's disease identifies new risk loci and implicates Abeta, tau, immunity and lipid processing. Nat. Genet. 51, 414-430 (2019).

36. Consortium, G. T. Human genomics. The Genotype-Tissue Expression (GTEx) pilot analysis: multitissue gene regulation in humans. Science $\mathbf{3 4 8}, 648-660$ (2015).

37. Trabzuni, D. et al. Quality control parameters on a large dataset of regionally dissected human control brains for whole genome expression studies. J. Neurochem. 119, 275-282 (2011).

38. Westmark, C. J. et al. Reversal of fragile $X$ phenotypes by manipulation of AbetaPP/Abeta levels in Fmr1KO mice. PLoS One 6, e26549 (2011).

39. Hong, L. et al. Quantification of the binding properties of $\mathrm{Cu} 2+$ to the amyloid beta peptide: coordination spheres for human and rat peptides and implication on Cu2+-induced aggregation. J. Phys. Chem. B 114, 11261-11271 (2010).

40. De Strooper, B. et al. Production of intracellular amyloid-containing fragments in hippocampal neurons expressing human amyloid precursor protein and protection against amyloidogenesis by subtle amino acid substitutions in the rodent sequence. EMBO J. 14, 4932-4938 (1995).

41. $\mathrm{Xu}, \mathrm{G}$. et al. Murine Abeta over-production produces diffuse and compact Alzheimer-type amyloid deposits. Acta Neuropathol. Commun. 3, 72 (2015).
42. Fabregat, A. et al. The reactome pathway knowledgebase. Nucleic Acids Res. 46, D649-D655 (2018)

43. Li, X. et al. Phosphorylated eukaryotic translation factor $4 \mathrm{E}$ is elevated in Alzheimer brain. Neuroreport 15, 2237-2240 (2004).

44. Furic, L. et al. elF4E phosphorylation promotes tumorigenesis and is associated with prostate cancer progression. Proc. Natl. Acad. Sci. USA 107, 14134-14139 (2010).

45. Chu, J., Cajal, S. R. Y., Sonenberg, N. \& Pelletier, J. Eukaryotic initiation factor 4Fsidestepping resistance mechanisms arising from expression heterogeneity. Curr. Opin. Genet. Dev. 48, 89-96 (2018).

46. Ueda, T., Watanabe-Fukunaga, R., Fukuyama, H., Nagata, S. \& Fukunaga, R. Mnk2 and Mnk1 are essential for constitutive and inducible phosphorylation of eukaryotic initiation factor 4E but not for cell growth or development. Mol. Cell. Biol. 24, 6539-6549 (2004).

47. Pyronnet, S. et al. Human eukaryotic translation initiation factor 4G (elF4G) recruits mnk1 to phosphorylate elF4E. EMBO J. 18, 270-279 (1999).

48. McKendrick, L., Morley, S. J., Pain, V. M., Jagus, R. \& Joshi, B. Phosphorylation of eukaryotic initiation factor $4 \mathrm{E}$ (elF4E) at Ser209 is not required for protein synthesis in vitro and in vivo. Eur. J. Biochem. 268, 5375-5385 (2001).

49. Richter, J. D. \& Sonenberg, N. Regulation of cap-dependent translation by elF4E inhibitory proteins. Nature 433, 477-480 (2005).

50. Hanger, D. P., Betts, J. C., Loviny, T. L., Blackstock, W. P. \& Anderton, B. H. New phosphorylation sites identified in hyperphosphorylated tau (paired helical filament-tau) from Alzheimer's disease brain using nanoelectrospray mass spectrometry. J. Neurochem. 71, 2465-2476 (1998).

51. Yamamoto, H. et al. Phosphorylation of tau at serine 416 by $\mathrm{Ca} 2+$ /calmodulindependent protein kinase II in neuronal soma in brain. J. Neurochem. 94 1438-1447 (2005)

52. Ghosh, A. \& Giese, K. P. Calcium/calmodulin-dependent kinase II and Alzheimer's disease. Mol. Brain 8, 78 (2015).

53. Plattner, F., Angelo, M. \& Giese, K. P. The roles of cyclin-dependent kinase 5 and glycogen synthase kinase 3 in tau hyperphosphorylation. J. Biol. Chem. 281, 25457-25465 (2006).

54. Pretorius, E. et al. Both lipopolysaccharide and lipoteichoic acids potently induce anomalous fibrin amyloid formation: assessment with novel Amytracker stains. J. R. Soc. Interface 15, (2018).

55. Braak, H. \& Braak, E. Neuropathological stageing of Alzheimer-related changes. Acta Neuropathol. 82, 239-259 (1991).

56. Khan, U. A. et al. Molecular drivers and cortical spread of lateral entorhinal cortex dysfunction in preclinical Alzheimer's disease. Nat. Neurosci. 17, 304-311 (2014).

57. Stranahan, A. M. \& Mattson, M. P. Selective vulnerability of neurons in layer II of the entorhinal cortex during aging and Alzheimer's disease. Neural Plast. 2010, 108190 (2010).

58. Li, C. \& Gotz, J. Somatodendritic accumulation of Tau in Alzheimer's disease is promoted by Fyn-mediated local protein translation. EMBO J. 36, 3120-3138 (2017).

59. Kobayashi, S., Tanaka, T., Soeda, Y., Almeida, O. F. X. \& Takashima, A. Local somatodendritic translation and hyperphosphorylation of tau protein triggered by AMPA and NMDA receptor stimulation. EBioMedicine 20, 120-126 (2017).

60. Hipp, M. S., Kasturi, P. \& Hartl, F. U. The proteostasis network and its decline in ageing. Nat. Rev. Mol. Cell Biol. 20, 421-435 (2019).

61. Itagaki, S., McGeer, P. L., Akiyama, H., Zhu, S. \& Selkoe, D. Relationship of microglia and astrocytes to amyloid deposits of Alzheimer disease. J. Neuroimmunol. 24, 173-182 (1989).

62. Wyss-Coray, T. et al. Adult mouse astrocytes degrade amyloid-beta in vitro and in situ. Nat. Med. 9, 453-457 (2003).

63. Abekhoukh, S. et al. New insights into the regulatory function of CYFIP1 in the context of WAVE- and FMRP-containing complexes. Dis. Model Mech. 10, 463-474 (2017).

64. Sasaguri, $H$. et al. APP mouse models for Alzheimer's disease preclinical studies. EMBO J. 36, 2473-2487 (2017)

65. Braak, H. \& Braak, E. Alzheimer's disease affects limbic nuclei of the thalamus Acta Neuropathol. 81, 261-268 (1991).

66. Youn, Y. C. et al. (11)C-PIB PET imaging reveals that amyloid deposition in cases with early-onset Alzheimer's disease in the absence of known mutations retains higher levels of PIB in the basal ganglia. Clin. Inter. Aging 12, 1041-1048 (2017). 
67. Aggleton, J. P., Pralus, A., Nelson, A. J. \& Hornberger, M. Thalamic pathology and memory loss in early Alzheimer's disease: moving the focus from the medial temporal lobe to Papez circuit. Brain 139, 1877-1890 (2016).

68. Saito, T. et al. Humanization of the entire murine Mapt gene provides a murine model of pathological human tau propagation. J. Biol. Chem. 294, 12754-12765 (2019).

69. Arnold, S. E., Hyman, B. T., Flory, J., Damasio, A. R. \& Van Hoesen, G. W. The topographical and neuroanatomical distribution of neurofibrillary tangles and neuritic plaques in the cerebral cortex of patients with Alzheimer's disease. Cereb. Cortex. 1, 103-116 (1991)

70. Garcia-Esparcia, P. et al. Altered mechanisms of protein synthesis in frontal cortex in Alzheimer disease and a mouse model. Am. J. Neurodegener. Dis. 6 , 15-25 (2017).
71. Ohno, M. PERK as a hub of multiple pathogenic pathways leading to memory deficits and neurodegeneration in Alzheimer's disease. Brain Res. Bull. (2017).

72. Brothers, H. M., Gosztyla, M. L. \& Robinson, S. R. The physiological roles of amyloid-beta peptide hint at new ways to treat Alzheimer's disease. Front. Aging Neurosci. 10, 118 (2018).

73. Arevalo, M. A., Roldan, P. M., Chacon, P. J. \& Rodriguez-Tebar, A. Amyloid beta serves as an NGF-like neurotrophic factor or acts as a NGF antagonist depending on its concentration. J. Neurochem. 111, 1425-1433 (2009).

74. Genheden, M. et al. BDNF stimulation of protein synthesis in cortical neurons requires the MAP kinase-interacting kinase MNK1. J. Neurosci. 35, 972-984 (2015).

75. Austen, M., Geese, M. \& Schneider, M. Use of Mnk inhibitors for the treatment of Alzheimer's disease. United States patent. (2010). 